\title{
Design of Uniformly Most Powerful Alphabets for HDF 2-Way Relaying Employing Non-Linear Frequency Modulations
}

\author{
Miroslav Hekrdla* and Jan Sykora
}

\begin{abstract}
Hierarchical-Decode-and-Forward is a promising wireless-network-coding-based 2-way relaying strategy due to its potential to operate outside the classical multiple-access capacity region. Assuming a practical scenario with channel state information at the receiver and no channel adaptation, there exist modulations and exclusive codes for which even non-zero channel parameters (denoted as catastrophic) cause zero hierarchical minimal distance significantly degrading its performance. In this work, we state that non-binary linear alphabets cannot avoid these parameters and some exclusive codes even imply them; contrary XOR does not. We define alphabets avoiding all catastrophic parameters and reaching its upper bound on minimal distance for all parameter values (denoted uniformly most powerful (UMP)). We find that binary, non-binary orthogonal and bi-orthogonal modulations are UMP. We optimize scalar parameters of FSK (modulation index) and full-response CPM (frequency pulse shape) to yield UMP alphabets.
\end{abstract}

\section{Introduction}

Cooperative communication in wireless relay networks can potentially be of great benefit, offering several gains that may decrease required transmission power, increase the system capacity, improve the cell coverage or interference mitigation while balancing the quality of service and keeping relatively low deployment costs [1]. Consequently, future wireless networks are envisaged to include the cooperative relaying techniques. However, it also brings several new challenging problems such as the extra resources (e.g. frequency or time slots) taken for interference-free relay traffic when considering practical halfduplex constraints (each node cannot send and receive at the same time). This is well demonstrated in the simplest cooperative network - 2-way relay channel (2-WRC) comprising two terminals $A$ and $B$ bidirectionally communicating between themselves via a supporting relay $\mathrm{R}$. Traditional protocols avoiding interference require 4 stages for every packet exchange (Figure 1a). Employing the advent of network coding [2] and wireless broadcast medium [3], the communication is more effective and

\footnotetext{
* Correspondence: miroslav.hekrdla@fel.cvut.cz

Faculty of Electrical Engineering, Department of Radio Engineering, Czech Technical University in Prague, Technicka 2, 16627 Praha 6, Czech Republic
}

reduces to 3 stages, Figure 1b). The first two stages of $3-$ stage protocols can be considered as a multiple-access (MAC) channel with orthogonally separated sources. Generally, setting the terminal rates to be within the MAC capacity region, we can reliably perform MAC in a single stage, resulting in a 2-stage protocol. Direct estimation of network coded data from signal interference is very promising due to its ability to operate outside this MAC capacity region [4]. This strategy appears under the name denoise-and-forward (DNF) or physical layer network coding [5]. Usually, a general term wireless network coding (WNC) is used to stress the fact that network coding-like operations are done in the wireless domain at the physical layer. Instead of DNF, we rather use generic term hierarchical-decode-and-forward (HDF) strategy, which is better suited specially for more complicated multisource networks [6].

HDF consists of MAC stage when both terminals transmit simultaneously to the relay with exclusively coded data decoding and broadcast $(\mathrm{BC})$ stage when the relay broadcasts the exclusively coded data, Figure 1c). The exclusive code (XC) permits message decoding at the terminals using their own messages serving as a complementary-side information [7].
(C) 2011 Hekrdla and Sykora; licensee Springer. This is an Open Access article distributed under the terms of the Creative Commons Attribution License (http://creativecommons.org/licenses/by/2.0), which permits unrestricted use, distribution, and reproduction in any medium, provided the original work is properly cited. 


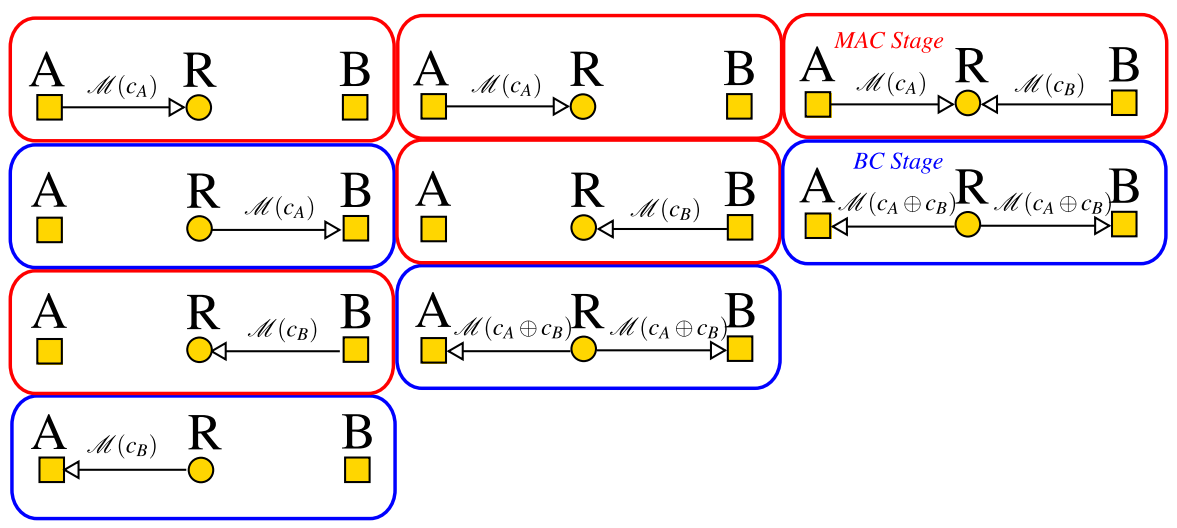

a) 4-stage

b) 3-stage

c) 2-stage

Figure 1 Basic division of 2-way relaying protocols.

HDF performance in MAC stage, assuming fading channel with channel state information at the receiver side (CSIR), is unavoidably parametric. There are some modulation alphabets (e.g., QPSK) for which even non-zero channel parameters (denoted as catastrophic) cause zero hierarchical minimal distance, which significantly degrades its performance. Adaptive extended-cardinality network coding [8] and adaptive precoding technique [9] were proposed to suppress this problem with channel parametrization. However, both techniques require some form of adaptation that might not be always available.

The aim of our paper is to introduce modulation alphabets and exclusive codes resistant to the problem of parametrization on condition of CSIR and no adaptation, similar to in [10]. We define a class of alphabets avoiding all catastrophic parameters and reaching its upper bound on minimal distance for all parameter values (denoted uniformly most powerful (UMP)). The papers [11], [12] are also related, restricting, however, on non-coherent (no CSIR) complex-orthogonal frequency shift keying (FSK) modulations.

The following contributions are provided:

1) Exclusive code $(X C)$ must fulfill certain conditions not to imply catastrophic parameters. Particularly, the $\mathrm{XC}$ matrix must be symmetric, and the same symbols must lie on its main diagonal. Bit-wise XOR operation obeys these conditions, and it is the only solution for binary and even quaternary alphabet. Hence, it is convenient to assume fixed XOR XC.

2) All non-binary linear modulations with one complex dimension (e.g., PSK, QAM) have inevitably catastrophic parameters and binary modulations not even fulfilling the UMP condition. It is shown that non-binary UMP alphabets require more than a single complex dimension.
3) Non-binary complex-orthogonal and non-binary complex bi-orthogonal modulations with XOR are UMP.

4) Non-linear frequency modulations FSK and fullresponse continuous phase modulation (CPM) naturally comprise multiple complex dimensions needed to obey UMP condition. We optimize a scalar parameters of FSK (modulation index) and full-response CPM (frequency pulse shape) to yield UMP alphabets. We find that a lower modulation index (proportional to bandwidth) than that leading to complex-orthogonal alphabet fulfills UMP condition. Numerical simulations conclude that the considered frequency modulations do not have catastrophic parameters and perform close to the utmost UMP alphabets which however require more bandwidth.

\section{System model}

\section{A. Constellation space model and used notation}

Let both terminals $A$ and $B$ in 2-WRC use the same modulation alphabet $\mathscr{A}$ with cardinality $|\mathscr{A}|=M_{\mathcal{C}}$ to be strictly a power of two. We suppose that the alphabet is formed by complex arbitrary-dimensional baseband signals in the constellation space $\mathscr{A}=\left\{\mathbf{s}_{c_{T}}\right\}_{\mathcal{C}_{T}=0}^{M_{c}-1} \subset \mathbb{C}^{N_{S}}$, where symbol $c_{T} \in \mathbb{Z}_{M_{c}}=\left\{0,1, \ldots, M_{c}-1\right\}$ denotes a data symbol transmitted by terminal $T \in\{A, B\}$ and $N_{s}$ denotes the signal dimensionality. Linear modulations (e.g., PSK, QAM) have single complex dimension, i.e., $N_{s}=1$ and their constellation vectors are complex scalars $s_{c_{t}} \in \mathbb{C}$. Later in this paper, we will use non-linear frequency modulations FSK and full-response CPM, which are multidimensional and its dimensionality is $N_{s}=M_{c}$; the constellation space vectors are consequently $\mathbf{s}_{c_{T}} \in \mathbb{C}^{N_{S}}$. Without loss of generality, we assume memoryless constellation mapper $\mathscr{M}$ such that it directly corresponds to the signal indexation, $\mathbf{s}_{c_{T}}=\mathscr{M}\left(c_{T}\right)$. 


\section{B. Model assumptions}

We assume a time-synchronized scenario with full CSIR, which is obtained, for example, by preceding tracking of pilot signals. The synchronization issues are beyond the scope of this paper, and interested reader may see e.g. [13], [14] for further details. We restrict ourselves that adaptive techniques are not available either due to the missing feedback channel, increased system complexity, or unfeasible channel dynamics.

We consider per-symbol relaying (avoiding delay induced at the relay) and no channel coding, which however can be additionally concatenated with our scheme [15].

\section{Hierarchical-decode-and-forward strategy}

HDF strategy in 2-WRC consists of two stages, see Figure 2.

In the first MAC stage, both terminals $A$ and $B$ transmit simultaneously to the relay in the interfering manner, see Figure 3.

The received composite (interfering) signal is

$$
\mathbf{x}=h_{A} \mathbf{s}_{c_{A}}+h_{B} \mathbf{s}_{c_{B}}+\mathbf{w},
$$

where $\mathbf{w}$ is complex AWGN with variance $2 N_{0}$ per complex dimension, and the channel parameters $h_{A}$ and $h_{B}$ are frequency-flat complex Gaussian random variables with unit variance and Rayleigh/Rician distributed envelope. The Rician factor $K$ is defined as a power ratio between stationary and scattered components. We assume that the channel parameters $h_{A}, h_{B}$ are known to $R$.

Subsequently, the relay decodes exclusively coded data symbol $c_{A B}=c_{A} \oplus c_{B}$ from interfering signal (1). Operation $\oplus$ is a network coding-like exclusive (invertible) operation, which incorporates data from multiple sources via a principle of exclusivity, see Section II-D for more details. We assume a minimal cardinality exclusive operation, i.e. cardinality of $c_{A B}$ alphabet is $M_{c}$ [7]. We suppose an approximated nearest neighbor twostep decoding [16]: estimate of exclusively coded data

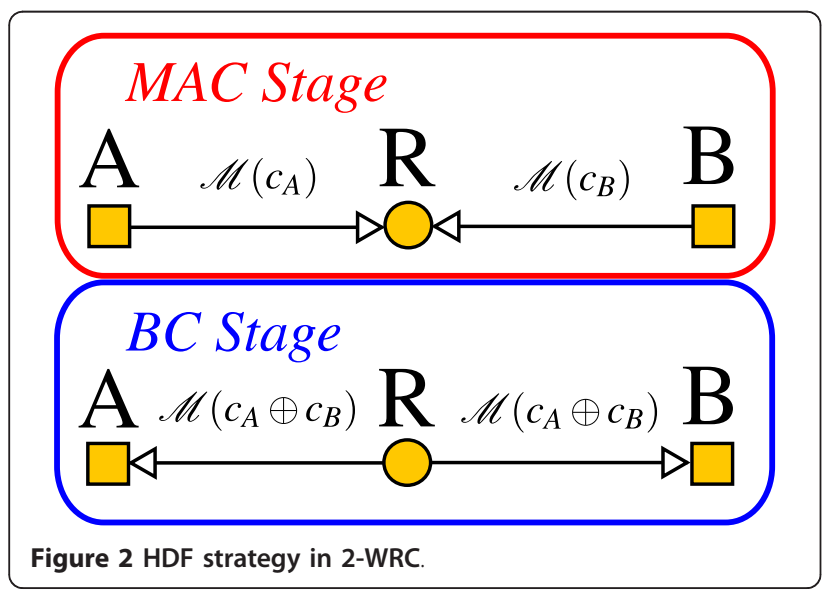

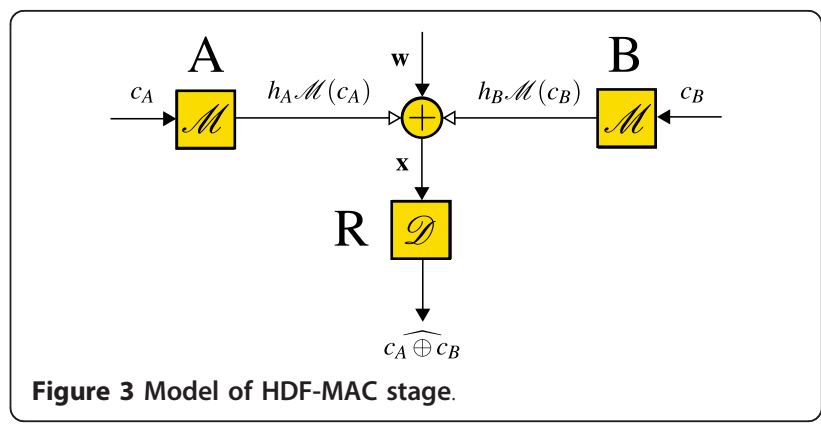

symbol $c_{A B}$ is obtained by joint maximum likelihood decoding

$$
\left[\hat{c}_{A}, \hat{c}_{B}\right]=\arg \min _{\left[c_{A}, c_{B}\right]}\left\|\mathbf{x}-h_{A} \mathbf{s}_{c_{A}}-h_{B} \mathbf{s}_{c_{B}}\right\|^{2}
$$

followed by exclusive encoding $\hat{c}_{A B}=\hat{c}_{A} \oplus \hat{c}_{B}$; by, $\|\star\|$ 2 , we denote the squared vector norm.

In the $\mathrm{BC}$ stage, $R$ broadcasts exclusive symbol $c_{A B}$, which is sufficient for successful decoding. Particularly, the terminal $A$ obtains desired data symbol $c_{B}$ with knowledge of $c_{A B}$ and its own data $c_{A}$ as $c_{B}=c_{A B} \ominus c_{A}$, where $\ominus$ denotes an inverse operation to exclusive coding and vice versa for $B$.

In this paper, we entirely focus on the MAC stage, which dominates the error performance, rather than $\mathrm{BC}$ stage due to the additional multiple-access interference [16].

\section{Exclusive coding}

We are aware that the term network coding is often used [5], but we rather propose the term exclusive coding to point out some important differences. Particularly, wellknown network coding is related to the link-layer techniques, and it is often assumed as a linear commutative operation with minimal cardinality of the output alphabet. Here, we require only existence of inversion, which we refer as an exclusivity. $\mathrm{XC}$ is regarded as an operation not necessarily commutative but with existence of inversion, i.e., a group. The XC operation can be well described by a matrix formed by exclusively coded symbols placed on a position corresponding to relevant $c_{A}$ and $c_{B}$. We denote this matrix as an exclusive code matrix $\mathrm{XC}$ given by $c_{A} \oplus c_{B}=[\mathrm{XC}]_{c_{A}, c_{B}}$. For example, the matrices

$$
\begin{aligned}
& \mathbf{X C}_{\text {XOR }}=\left[\begin{array}{ll}
0 & 1 \\
1 & 0
\end{array}\right] \\
& \mathbf{X C}_{\text {ModSum }}=\left[\begin{array}{llll}
0 & 1 & 2 & 3 \\
1 & 2 & 3 & 0 \\
2 & 3 & 0 & 1 \\
3 & 0 & 1 & 2
\end{array}\right]
\end{aligned}
$$




$$
\mathrm{XC}_{\text {BitXOR }}=\left[\begin{array}{llll}
0 & 1 & 2 & 3 \\
1 & 0 & 3 & 2 \\
2 & 3 & 0 & 1 \\
3 & 2 & 1 & 0
\end{array}\right]
$$

define binary XOR, quaternary modulo sum and quaternary bit-wise XOR operation, respectively. We will use often short 'XOR' to denote bit-wise XOR exclusive code. The notation resembles Sudoku game where in each row and each column, every element can appear only once. Exclusively coded symbols in XC matrix may take different values, the only demand is the existence of inversion $\ominus$. For instance, the inversion exists also if we replace one symbol with a new not yet introduced, which extends the cardinality of exclusively coded symbols $c_{A B}$. We assume the XC matrix in a standard form where the first row is in increasing order starting from zero.

We restrict ourselves on the minimal cardinality $\mathrm{XC}\left(c_{A B} \in \mathbb{Z}_{M_{c}}\right)$ in order to avoid redundancy in the BC stage. It is interesting that the number of all distinct $\mathrm{XC}$ matrices with the minimal cardinality (a.k.a. Latin squares) grows very fast with alphabet size [17] as depicted in Table 1.

\section{E. Parametric Hierarchical constellation}

Since the relay has CSIR, we will conveniently introduce a model of hierarchical constellation, which uses instead of $h_{A}, h_{B}$ only one complex parameter $\alpha$ always $|\alpha| \leq 1$ [18].

The useful received signal (1) can be normalized by $h_{A}$

$$
\mathbf{x}^{\prime}=\mathbf{x} / h_{A}=\mathbf{s}_{c_{A}}+\alpha \mathbf{s}_{c_{B}}+\mathbf{w}^{\prime},
$$

where $\alpha=h_{B} / h_{A} \in \mathbb{C}$ and $\left.E|| \mathbf{w}^{\prime}||^{2}\right]=2 N_{0} N_{S} /\left|h_{A}\right|^{2}$ or by $h_{B}$

$$
\mathbf{x}^{\prime \prime}=\mathrm{x} / h_{B}=1 / \alpha \mathbf{s}_{c_{A}}+\mathbf{s}_{c_{B}}+\mathbf{w}^{\prime \prime} \text {, }
$$

with $E\left[|| \mathbf{w}^{\prime \prime}||^{2}\right]=2 N_{0} N_{S} /\left|h_{B}\right|^{2}$ where operator $E[\star]$ denotes the statistical expectation. By adaptive switching between (6) and (7), we ensure that $|\alpha|$ or $|1 / \alpha|$ is always lower or equal to one. The decoding processing (2) remains the same for both cases; therefore, the switching has only theoretical value that we need to focus on system performance only for $|\alpha| \leq 1$. The useful hierarchical (composite) signal is $u_{c_{A} c_{B}}(\alpha)=\mathbf{s}_{c_{A}}+\alpha \mathbf{s}_{c_{B}}$ or $u_{c_{A} c_{B}}(\alpha)=\alpha \mathbf{s}_{c_{A}}+\mathbf{s}_{c_{B}}$ according to $|\alpha|$; however, for both cases, useful parametric hierarchical signal at the relay is

Table 1 Number of minimal cardinality exclusive codes in the standard notation as a function of the alphabet size

\begin{tabular}{cccc}
\hline $\boldsymbol{M}_{\boldsymbol{c}}$ & $\mathbf{2}$ & $\mathbf{4}$ & $\mathbf{8}$ \\
\hline Number of $X C s$ & 1 & 24 & $\sim 10^{16}$ \\
\hline
\end{tabular}

$$
\mathbf{u}_{c_{A} c_{B}}(\alpha)=\mathbf{s}_{c_{A}}+\alpha \mathbf{s}_{c_{B}}, \quad|\alpha| \leq 1,
$$

because both terminals have the same $\mathscr{A}$.

III. Hierarchical minimal distance as a performance metric and catastrophic parameters.

\section{A. Hierarchical minimal distance}

Let us focus on the error performance in the MAC stage. Defining a symbol error $\partial_{A B} \neq c_{A B}$, the symbol error probability is well approximated by sum of weighted pairwise error probabilities. The pairwise error probability is a function of distance between signals corresponding to $c_{A B} \neq c_{A B}^{\prime}$ and the pairwise error probability dominating the performance for high signal-tonoise ratio (SNR) is a function of the minimal distance. In our case, the minimal distance is a minimal distance between hierarchical signals $\mathrm{u}_{\mathcal{C}_{A} c_{B}}$ and $\mathrm{u}_{\mathcal{C}^{\prime} \mathcal{C}_{B^{\prime}}}$ with different XC symbols,

$$
d_{\min }^{2}(\alpha)=\min _{c_{A} \oplus c_{B} \neq c_{A^{\prime}} \oplus c_{B^{\prime}}}\left\|\mathbf{u}_{c_{A} c_{B}}(\alpha)-\mathbf{u}_{c_{A^{\prime}} c_{B^{\prime}}}(\alpha)\right\|^{2}
$$

and we will call it a hierarchical minimal distance; when it is clear, we omit the attribute hierarchical.

Note that the minimal distance is given not only by modulation alphabet but also by XC operation. In general, the minimal distance is parametrized by $\alpha$ and so is the error performance.

Remark 1. Facing the fact that the hierarchical constellation is randomly parametrized, we start investigation with the simplification that the error performance is given solely by minimal distance. We are aware that this is a rough approximation, since the minimal distance is relevant performance metric only asymptotically (as SNR $\rightarrow \infty$ ) and the error curves are linearly proportional also to the number of signal pairs having the minimal distance.

\section{B. Catastrophic parameters and paper motivation}

In the preceding section, we have seen that the HDFMAC stage with CSIR has parametric minimal distance (asymptotic performance). For some modulation alphabets and exclusive codes, there exist such non-zero parameters (called catastrophic), which yields even zero minimal distance. This problem is well demonstrated, for example, by QPSK and complex-orthonormal QFSK (modulation index $\kappa=1$ ) modulation with XOR (5). Minimal distance of QPSK is depicted in Figure 4a indicating several catastrophic parameters e.g. for $\alpha_{\text {cat }}=j$. On the contrary, numerical evaluation of QFSK with $\kappa=1$, see Figure $4 \mathrm{~b}$, seems to have minimal distance parabolically dependent on $|\alpha|$ as

$$
d_{\min }^{2}(\alpha)=2|\alpha|^{2}
$$




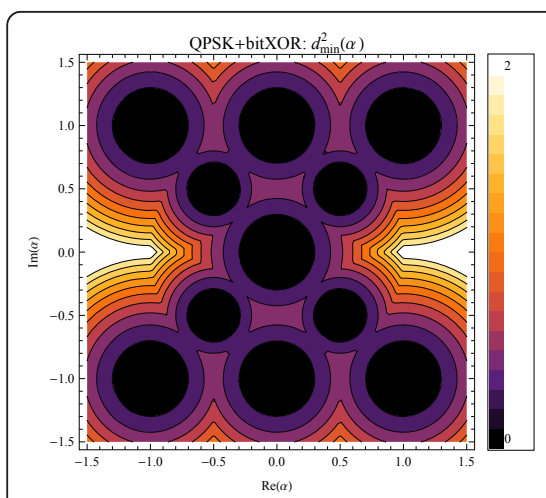

(a)

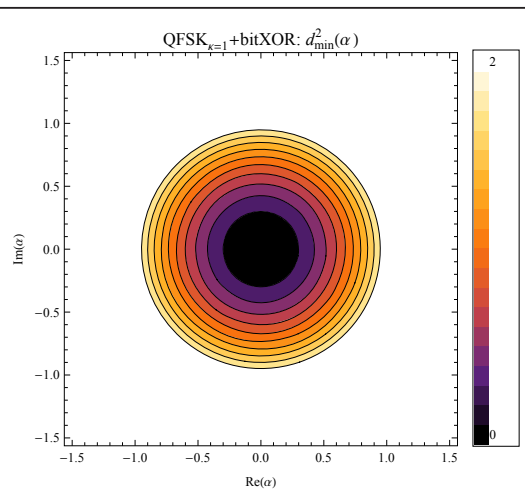

(b)

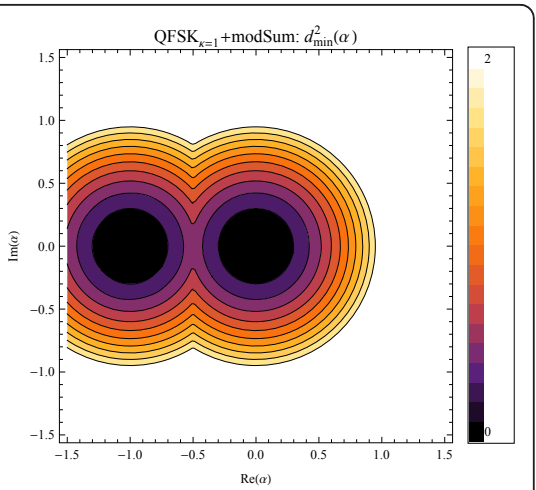

(c)

Figure 4 Parametric minimal distance of QPSK and XOR (a) - for some non-zero parameters we expect poor performance. QFSK with $\kappa=$ 1 and XOR (b) is robust to parametrization - is uniformly most powerful (UMP). QFSK with $\kappa=1$ and modulo sum XC (c) is not UMP due to the poor performance for the parameter -1 .

It has no catastrophic parameters, and therefore, it is robust to the parametrization. Zero distance for $\alpha=0$ is expected because it means that one of the channel is relatively zero. This paper focuses on the design of alphabets and XCs like this. We demonstrate by Figure 4c with QFSK $\kappa=1$ and modulo sum XC (4) that not only modulation alphabet, but also exclusive code influence the parameter robustness.

Before we state the core idea of UMP alphabet, let us precisely define catastrophic parameters and state a couple of important lemmas based on them.

Definition 2 (Catastrophic parameters). A catastrophic parameter is such a non-zero parameter $\alpha_{\text {cat }}$ that forces two hierarchical signals corresponding to different XC symbols to the same point (equivalently, it indicates zero minimal distance). In our notation, for some $\alpha_{\text {cat }} \neq 0$, there exists $c_{A} \oplus c_{B} \neq c_{A^{\prime}} \oplus c_{B^{\prime}}$ that

$$
\left\|\mathbf{u}_{c_{A} c_{B}}\left(\alpha_{\text {cat }}\right)-\mathbf{u}_{c_{A^{\prime}} c_{B^{\prime}}}\left(\alpha_{\text {cat }}\right)\right\|^{2}=0 .
$$

\section{Exclusive code not implying catastrophic parameters}

This section shows that XC must fulfill certain conditions not to imply catastrophic parameters regardless of the modulation alphabet. This reduces the number of XCs, see Table 1 involved in search for alphabets and $\mathrm{XCs}$ robust to the parametrization. The conditions are derived again in order to avoid catastrophic parameters.

Theorem 3. A matrix of XC with different symbols on the main diagonal implies $\alpha_{\mathrm{cat}}=-1$ and XC matrix which is not symmetric over the main diagonal has $\alpha_{\mathrm{cat}}=1$ regardless of modulation.

Proof: Let two hierarchical signals correspond to the XC matrix main diagonal, $c_{A}=c_{B}, c_{A}{ }^{\prime}=c_{B}$ ' i.e. $\mathbf{u}_{c_{A} c_{A}}$, $\mathbf{u}_{c^{\prime}{ }^{\prime} c^{\prime}, c_{A}} \neq c_{A}{ }^{\prime}$ and their XC symbols are different $c_{A} \oplus$ $c_{A} \neq c_{A}^{\prime} \oplus c_{A}^{\prime}$. Equation (11) is then

$$
\| \mathbf{s}_{c_{A}}-\mathbf{s}_{c_{A^{\prime}}}+\left.\alpha\left(\mathbf{s}_{c_{A}}-\mathbf{s}_{c_{A^{\prime}}}\right)\right|^{2}=|1+\alpha|^{2}|| \mathbf{s}_{c_{A}}-\left.\mathbf{s}_{c_{A^{\prime}}}\right|^{2}
$$

and $\alpha_{\text {cat }}=-1$., which is similar to for non-symmetric XC matrix. Assume $\mathrm{u}_{c_{A} c_{B}}, u_{c_{B} c_{A}}$ with $c_{A} \oplus c_{B} \neq c_{B} \oplus c_{A}$, Equation (11) is

$$
\|\left|\mathbf{s}_{c_{A}}-\mathbf{s}_{c_{B}}+\alpha\left(\mathbf{s}_{c_{B}}-\mathbf{s}_{c_{A}}\right)\right|^{2}=|1-\alpha|^{2}|| \mathbf{s}_{c_{A}}-\left.\mathbf{s}_{c_{B}}\right|^{2}
$$

and $\alpha_{\text {cat }}=1$. We conclude that XC matrix should be symmetric with the same code symbols on its main diagonal.

Remark 4 (Suitability of bit-wise XOR XC). XOR fulfills these conditions, and it is the only solution for binary and even quaternary alphabet (unfortunately it is not the only choice for e.g. octal alphabet) [17]. Once we fix XC (at least for binary and quaternary case), the only thing that influences the parameter robustness is the modulation alphabet. Therefore, from now on, we assume $\oplus$ is $X O R$ for all cases and we relate the parametrization robustness only with particular modulation alphabets.

\section{Non-binary linear modulations are catastrophic}

In this section, we demonstrate that any non-binary linear modulation can never avoid catastrophic parameters, as we have seen particularly for QPSK, Figure 4a.

Lemma 5. Non-binary linear modulations unavoidably have catastrophic parameters.

Proof: Linear modulations like QAM and PSK have dimensionality $N_{s}=1$ and signals in the constellation space are $s_{A}, s_{B} \in \mathbb{C}$. Considering hierarchical signals $u_{c^{\prime}{ }^{\prime} c^{\prime}}, u_{c_{A}{ }^{\prime} c_{B}{ }^{\prime}}$ with $c_{A} \oplus c_{B} \neq c_{A}{ }^{\prime} \oplus c_{B}{ }^{\prime}$ and symbols not being in the same row and column of XC matrix $\left(c_{A} \neq\right.$ $\left.c_{A}{ }^{\prime}, c_{A}{ }^{\prime} \neq c_{B}{ }^{\prime}\right)$, there exists such a parameter that

$$
\begin{gathered}
u_{C_{A} c_{B}}\left(\alpha^{\prime}\right)=u_{C^{\prime} A c^{\prime} B}\left(\alpha^{\prime}\right), \\
s_{C_{A}}+\alpha^{\prime} s_{C_{B}}=s_{C_{A^{\prime}}}+\alpha^{\prime} s_{C_{B}{ }^{\prime}},
\end{gathered}
$$


this parameter equals to $\alpha^{\prime}=\left(s_{c_{A^{\prime}}}-s_{c_{A}}\right) /\left(s_{c_{B}}-s_{c_{B^{\prime}}}\right)$. Binary alphabets are excluded from consideration while its different hierarchical signals always lie in the same row or column of the XC matrix.

Since we assume channel model switching, it would be a catastrophic parameter if it was $\left|\alpha^{\prime}\right| \leq 1$, but as we discussed in the previous section, the XC matrix should be symmetric and so, also for symmetric signals $u_{c_{B} C_{A}}, u_{c_{B}{ }^{\prime} c_{A}}$, there exists a parameter $\alpha^{\prime \prime}$ such

$$
\begin{gathered}
u_{c_{B} C_{A}}\left(\alpha^{\prime \prime}\right)=u_{c_{B}{ }^{\prime} A^{\prime}}\left(\alpha^{\prime \prime}\right), \\
s_{C_{B}}+\alpha^{\prime \prime} s_{C_{A}}=s_{C_{B}{ }^{\prime}}+\alpha^{\prime \prime} s_{C_{A^{\prime}},}
\end{gathered}
$$

$\alpha^{\prime \prime}=1 / \alpha^{\prime}$. Hence, the catastrophic parameter equals to $\alpha^{\prime}$ or $\alpha^{\prime \prime}$, whether its absolute value is lower or equal to one.

\section{Uniformly most powerful alphabet}

Inspired by previous sections, we define a class of alphabets with hierarchical minimal distance of the form like in (10) avoiding all catastrophic parameters and being robust to the channel parametrization. We will show that the form (10) corresponds to alphabets reaching the minimal distance upper-bound for all parameter values.

\section{A. Minimal distance upper-bound}

Lemma 6. Minimal distance of any alphabet is upperbounded by

$$
d_{\min }^{2}(\alpha) \leq|\alpha|^{2} \delta_{\min ^{\prime}}^{2}
$$

where $\delta_{\min }^{2}$ is a minimal distance of a single (non-hierarchical) modulation alphabet; $\delta_{\min }^{2}=\min _{c_{A} \neq c_{\Lambda^{\prime}}}\left\|\mathbf{s}_{c_{A}}-\mathbf{s}_{c_{A^{\prime}}}\right\|^{2}$ and $c_{A}, c_{A}{ }^{\prime} \in \mathbb{Z}_{M_{c} \cdot}$.

Proof: We obtain the upper bound by evaluating minimum operator only along the hierarchical signals corresponding to a single row of XC matrix (it means for $\left.c_{A}=c_{A}{ }^{\prime}\right)$,

$$
d_{\min }^{2}(\alpha) \leq \min _{c_{B} \neq c_{B}^{\prime}},\left\|\mathbf{u}_{c_{A} c_{B}}-\mathbf{u}_{c_{A} c_{B^{\prime}}}\right\|^{2}=\min _{c_{B} \neq \mathcal{C}_{B^{\prime}}},|\alpha|^{2}|| \mathbf{s}_{c_{B}}-\mathbf{s}_{c_{B^{\prime}}{ }^{\prime}} \|^{2} .
$$

Since we do not evaluate the minimum operator along the all possible hierarchical signal differences, we need to use inequality in (17). The minimum evaluation along a single column of XC matrix $\left(c_{B}=c_{B}^{\prime}\right.$ in (9)) yields

$$
\min _{c_{A} \neq c_{A^{\prime}}}\left\|\mathbf{u}_{c_{A} c_{B}}-\mathbf{u}_{c_{A^{\prime}} c_{B}}\right\|^{2}=\min _{c_{A} \neq c_{A^{\prime}}}\left\|\mathbf{s}_{c_{A}}-\mathbf{s}_{c_{A^{\prime}}}\right\|^{2}=\delta_{\min }^{2} \cdot
$$

As we are considering $|\alpha| \leq 1$, we conclude that the bound (16) is more tight than (18).

\section{B. UMP alphabet definition}

Definition 7. Uniformly most powerful (UMP) alphabets have hierarchical minimal distance reaching the upperbound (16) for all parameter values and it equals to

$$
d_{\min }^{2}(\alpha)=|\alpha|^{2} \delta_{\min ^{\prime}}^{2}, \forall \alpha \in \mathbb{C},|\alpha| \leq 1,
$$

where $\delta_{\min }^{2}=\min _{c_{A} \neq c_{c^{\prime}} \mid} \mid \mathbf{s}_{c_{A}}-\mathbf{s}_{c_{A^{\prime}}} \|^{2}$ and $c_{A}, c_{A}{ }^{\prime} \in \mathbb{Z}_{M_{c^{\prime}}}$ We restrict on $|\alpha| \leq 1$ due to the adaptive switching, Section II-E.

\section{UMP alphabet properties}

Lemma 8. It is important to stress that UMP alphabets do not have any catastrophic $\alpha_{\mathrm{cat}}$, and according to Lemma 5 and Remark 4, non-binary linear modulations are never UMP and all UMP alphabets are using XOR exclusive code.

Remark 9. Extended-cardinality XC as well as minimal cardinality XC have different code symbols in each XC matrix row (Sudoku principle) and thus the bound holds for extended-cardinality XC as well; particularly, for systems with adaptive XC [8]. In the other words, the performance of adaptive $\mathrm{XC}$ system cannot be better than of UMP alphabet if both are using alphabets with the same $\delta_{\min }^{2}$

Remark 10. Two properties influence good HDF performance, a) being UMP and b) having large minimal distance of individual constellations $\delta_{\text {min }}^{2}$. These properties can be interpreted as follows. The property b) is proportional to robustness to AWGN. The UMP condition a) (considering the upper-bound (16)) presents the best possible type of inevitable parametrization by $\alpha$.

Remark 11 (Parallel with UMP statistical tests). Simplifiedly matching error performance with minimal distance (Remark 1), we state that among all alphabets with identical $\delta_{\text {min }}^{2}$, the UMP alphabets have the best performance $\forall \alpha \in \mathbb{C}$. Based on this observation, we use the term UMP originally used in statistical detection theory due to the common principle. Composite hypothesis tests have parametrized PDFs and UMP detector, if exists, assuming knowledge of the instant value of the random parameter yields the best performance for all parameter values [19]. It resembles exactly our case, the likelihood function of joint $\left[c_{A}, c_{B}\right]$ detection is also parametrized (by $h_{A}, h_{B}$ ) [10] and assuming CSIR the optimal detector of UMP alphabets has the best performance for all parameter values.

\section{Binary modulation is UMP}

Evaluating formula (9) with respect to the binary XC matrix (3), we straightforwardly obtain

$$
d_{\min }^{2}(\alpha)=|\alpha|^{2} \delta_{\min ^{\prime}}^{2}
$$

where $\delta_{\min }^{2}=\left\|\mathbf{s}_{0}-\mathbf{s}_{1}\right\|^{2}$. It means that binary alphabets are always UMP regardless of the particular alphabet. Considering Remark 10, the optimal binary UMP alphabet is BPSK which maximizes $\delta_{\min }^{2}$. 


\section{E. Non-binary orthonormal modulation is UMP}

We have seen in Figure 4b that complex-orthonormal QFSK is UMP. This holds in general which describes the following lemma.

Lemma 12. Complex-orthonormal modulation is UMP.

Remark 13. Before we prove the Lemma 12, it is convenient to introduce simplified UMP condition easier to verify.

The UMP condition (19) also implies that the minimum distance is formed by the hierarchical signal differences corresponding to the rows of XC matrix, see also the proof of Lemma 6. Therefore, if the squared norm of hierarchical signal differences with indices not being in the same row of XC matrix are always larger or equal than the bound,

$$
\left\|\mathbf{u}_{c_{A} c_{B}}-\mathbf{u}_{c_{A}{ }^{\prime} c_{B}{ }^{\prime}}\right\|^{2} \geq|\alpha|^{2} \delta_{\min ^{\prime}}^{2}
$$

for $\forall c_{A} \neq c_{A}{ }^{\prime}, c_{B} \neq c_{B}{ }^{\prime}, c_{A} \oplus c_{B} \neq c_{A}{ }^{\prime} \oplus c_{B}{ }^{\prime}$ and $\forall \alpha \in$ $\mathbb{C},|\alpha| \leq 1$, then (19) is fulfilled. Expanded left side of (21) is

$$
\|\left|\mathbf{s}_{c_{A}}-\mathbf{s}_{c_{A^{\prime}}}\right|^{2}+|\alpha|^{2}|| \mathbf{s}_{c_{B}}-\left.\mathbf{s}_{c_{B^{\prime}}}\right|^{2}+2 \Re\left\{\alpha^{*}\left\langle\mathbf{s}_{c_{A}}-\mathbf{s}_{c_{A^{\prime}}}, \mathbf{s}_{c_{B}}-\mathbf{s}_{c_{B}}\right\rangle\right\},
$$

where $\langle\star, \star\rangle$ denotes an inner product. Since inequality (22) must hold for all $\phi=\arg \alpha$, it must hold for the worst case $\phi_{c}$, where the part with inner product is minimal and (22) becomes

$$
\left.\left\|\mathbf{s}_{c_{A}}-\left.\mathbf{s}_{c_{A}}{ }^{\prime}\right|^{2}+|\alpha|^{2}|| \mathbf{s}_{c_{B}}-\mathbf{s}_{c_{B}{ }^{\prime}}\right\|^{2}-2|\alpha||| \mathbf{s}_{c_{A}}-\mathbf{s}_{c_{A^{\prime}},} \mathbf{s}_{c_{B}}-\mathbf{s}_{c_{B}{ }^{\prime}}\right\rangle \mid .
$$

This form of invariancy condition is easier to verify due to the presence of only one real variable $|\alpha|$.

Proof: Orthonormal modulation has all distances (as well as the minimal one) for $c_{A} \neq c_{A}{ }^{\prime}, c_{B} \neq c_{B}$ ' equal to 2 , then (23) simplifies to

$$
2+2|\alpha|^{2}-2|\alpha|\left|\left\langle\mathbf{s}_{c_{A}}-\mathbf{s}_{c_{A^{\prime}},} \mathbf{s}_{c_{B}}-\mathbf{s}_{{C_{B}}^{\prime}}\right\rangle\right| \geq 2|\alpha|^{2}
$$

which further adjusts to

$$
1 \geq|\alpha|\left|\left\langle\mathbf{s}_{c_{A}}-\mathbf{s}_{c_{A}{ }^{\prime}}, \mathbf{s}_{c_{B}}-\mathbf{s}_{c_{B}{ }^{\prime}}\right\rangle\right|
$$

While (25) must hold for any $|\alpha| \leq 1$, it requires to hold for critical $|\alpha|=1$. Here, a critical parameter is such a parameter that if the condition is fulfilled for that one, then it is fulfilled for all other parameter values. Condition (25) with critical $|\alpha|=1$ is then

$$
\begin{aligned}
& 1 \geq\left|\left\langle\mathbf{s}_{C_{A}}-\mathbf{s}_{c_{A^{\prime}}}, \mathbf{s}_{C_{B}}-\mathbf{s}_{\mathcal{C}_{B}{ }^{\prime}}\right\rangle\right|= \\
& =\left|\left\langle\mathbf{s}_{c_{A}}, \mathbf{s}_{C_{B}}\right\rangle+\left\langle\mathbf{s}_{C_{A}{ }^{\prime}}, \mathbf{s}_{\mathcal{C}_{B}{ }^{\prime}}\right\rangle-\left\langle\mathbf{s}_{c_{A}}, \mathbf{s}_{c_{B}{ }^{\prime}}\right\rangle-\left\langle\mathbf{s}_{c_{A}{ }^{\prime}}, \mathbf{s}_{C_{B}}\right\rangle\right| .
\end{aligned}
$$

We prove (26) considering that any inner product of orthonormal modulation is either 0 or 1 . Equation (26) is fulfilled except for the case where the r.h.s. equals to 2 . It happens when $\left\langle\mathbf{s}_{\mathcal{C}_{A}}, \mathbf{s}_{\mathcal{C}_{B}}\right\rangle=1 \&\left\langle\mathbf{s}_{C_{A^{\prime}},}, \mathbf{s}_{C_{B^{\prime}}}\right\rangle=1 \&\left\langle\mathbf{s}_{C_{A^{A}}}, \mathbf{s}_{C_{B}{ }^{\prime}}\right\rangle=0$ $\&\left\langle\mathbf{s}_{C_{A^{\prime}},}, \mathbf{s}_{C_{B}}\right\rangle=0$ and when $\left\langle\mathbf{s}_{C_{A}}, \mathbf{s}_{C_{B}}\right\rangle=0 \quad \& \&\left\langle\mathbf{s}_{C_{A^{\prime}}{ }^{\prime}}, \mathbf{s}_{C_{B}{ }^{\prime}}\right\rangle=0$
$\& \&\left\langle\mathbf{s}_{C_{A}}, \mathbf{s}_{C_{B}}\right\rangle=1 \&\left\langle\mathbf{s}_{C_{A^{\prime}},}, \mathbf{s}_{C_{B}}\right\rangle=1$. Let us consider the first case, $\left\langle\mathbf{s}_{C_{A}}, \mathbf{s}_{C_{B}}\right\rangle=1 \&\left\langle\mathbf{s}_{C_{A^{\prime}}}, \mathbf{s}_{C_{B}}\right\rangle=1$ entails that $\mathbf{s}_{c_{A}}=\mathbf{s}_{C_{B}}$ $\& \mathbf{s}_{c_{A^{\prime}}}=\mathbf{s}_{c_{B}{ }^{\prime}}$ thus $c_{A}=c_{B} \& c_{A}{ }^{\prime}=c_{B}{ }^{\prime}$, which corresponds to hierarchical signals from the main diagonal of the $\mathrm{XC}$ matrix. Thus, using the XC code suitable for UMP, see Remark 4, this case is excluded. Similarly, the second condition $\&\left\langle\mathbf{s}_{C_{A}}, \mathbf{s}_{C_{B}{ }^{\prime}}\right\rangle=1 \&\left\langle\mathbf{s}_{c_{A^{\prime}}}, \mathbf{s}_{C_{B}}\right\rangle=1$ implies $c_{A}=c_{B}{ }^{\prime} \& c_{B}=$ $c_{A}$ ' and is excluded by $\mathrm{XC}$ with symmetrical $\mathrm{XC}$ matrix, again excluded by XOR.

\section{Design of ump frequency modulations}

In this section, we consider non-linear frequency modulations that naturally possess multidimensional alphabets, according to Lemma 5 needed to avoid catastrophic parameters. We will conclude that the considered frequency modulations avoid catastrophic parameters and are close to meet the UMP condition. We propose and use simple scalar alphabet parametrization easy to meet the UMP condition. Based on the error simulations, we will find that existence of catastrophic parameters is much more detrimental than not being UMP. In case of frequency modulations (without catastrophic parameters), UMP alphabets are important since according to Remark 11, they form a performance benchmark.

\section{A. UMP-FSK design}

1) FSK definition and basic properties: We assume the following unit energy FSK signals of one symbol duration

$$
s_{c}(t)=e^{j 2 \pi \kappa c \frac{t}{T_{s}}},
$$

where $t \in\left[0, T_{s}\right)$ is a temporal variable, $T_{s}$ is a symbol duration and $c \in \mathbb{Z}_{M_{c}}$ denotes a data symbol. Its constellation space alphabet is $N_{s}$-dimensional $\mathscr{A}=\left\{\mathbf{s}_{c}\right\}_{c=0}^{M_{c}-1} \subset \mathbb{C}^{N_{s}}$, where $N_{s}=M_{c}$. Its signal correlation as well as minimal distance is determined by modulation index $\kappa$ which also roughly corresponds to the occupied bandwidth [20]. It is well-known fact that FSK is complex-orthonormal for integer modulation index $\kappa \in \mathbb{N}$ and with minimal $\kappa=1$ is often used in non-coherent detection. In coherent detection (with CSIR), it has maximal minimal distance $\delta_{\min }^{2}=2$ for $\kappa=1 / 2$ also often denoted as minimum shift.

2) Design of UMP-QFSK modulation by index optimization: According to Lemma 12, FSK $\kappa=1$ is UMP. Yet, we try to answer a question whether full complexorthogonality is required to meet UMP. To investigate the non-orthogonal case, we assume $\kappa<1$, which also means a modulation roughly with narrower bandwidth, see more detailed discussion of bandwidth requirements in Section VI-C. 
Let us assume quaternary $M_{c}=4$ (binary is UMP regardless of alphabet, Section IV-D) FSK (QFSK) to consider this question where we optimize modulation index $\kappa$ to meet the UMP condition. The following lemma is true.

Lemma 14. QFSK $\kappa=5 / 6$ is UMP, see Figure 5b. The same approach may be used for any alphabet cardinality - the results for octal UMP-FSK require $\kappa=13 / 14$ which leads to a conjecture that $\kappa\left(M_{c}\right)=2 M_{c}-3 / 2 M_{c}-2$ is sufficient.

Proof: We have seen in Section IV-E that the condition implying UMP property (23) is

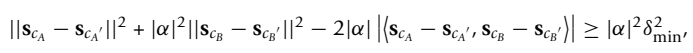

for $c_{A} \neq c_{A}{ }^{\prime}, c_{B} \neq c_{B}{ }^{\prime}, c_{A} \oplus c_{B} \neq c_{A}{ }^{\prime} \oplus c_{B}{ }^{\prime}$ and $\forall \alpha \in \mathbb{C}$, $|\alpha| \leq 1$. The following steps further adjust (28) to a suitable 2nd order polynomial form

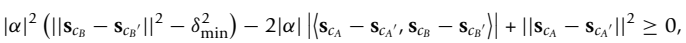

$$
\begin{aligned}
& |\alpha|^{2}-2|\alpha| \frac{\left|\left\langle\mathbf{s}_{c_{A}}-\mathbf{s}_{c^{\prime}}, \mathbf{s}_{c_{B}}-\mathbf{s}_{c_{B^{\prime}}}\right\rangle\right|}{\| \mathbf{s}_{c_{B}}-\left.\mathbf{s}_{c_{c^{\prime}}}\right|^{2}-\delta_{\text {min }}^{2}}+\frac{\left\|\mathbf{s}_{c_{A}}-\mathbf{s}_{c_{A^{\prime}}}\right\|^{2}}{\| \mathbf{s}_{c_{B}}-\left.\mathbf{s}_{c_{B}^{\prime}}\right|^{2}-\delta_{\min }^{2}} \geq 0, \\
& (|\alpha|-b)^{2}+c \geq 0,
\end{aligned}
$$

where auxiliary constants

$$
b=\left|\left\langle\mathbf{s}_{c_{A}}-\mathbf{s}_{c_{A^{\prime}}}, \mathbf{s}_{c_{B}}-\mathbf{s}_{c_{B^{\prime}}}\right\rangle\right| /\left\|\left\langle\mathbf{s}_{c_{B}}-\mathbf{s}_{c_{B}}\right\rangle\right\|^{2}-\delta_{\min }^{2} b \geq 0
$$

and $c=-b^{2}+\left\|\mathbf{s}_{c_{A}}-\mathbf{s}_{c_{A^{\prime}}}\right\|^{2} /\left\|\mathbf{s}_{c_{B}}-\mathbf{s}_{c_{B}{ }^{\prime}}\right\|^{2}-\delta_{\min }^{2}$ are not functions of $|\alpha|$. Thus, the condition (28) has a critical $|\alpha|$, which either equals to $b$ if $b \leq 1$ or limits value 1 if $b \geq 1$. In Figure 6 , we plot the constant $b$ for all indices $c_{A} \neq c_{A}{ }^{\prime}, c_{B} \neq c_{B}{ }^{\prime}, c_{A} \oplus c_{B} \neq c_{A}{ }^{\prime} \oplus c_{B}{ }^{\prime}$ for QFSK and XOR XC. We conclude that constant $b$ is always greater than 1 for roughly $\kappa \geq 0.3$. For practical purposes, we restrict on $\kappa \geq 1 / 2$ because then the minimal distance $\delta_{\min }^{2}$ is reasonably high. The restriction implies that constant $b \geq 1$ and so the critical $|\alpha|=1$. L.h.s of (28) for critical $|\alpha|=1$ are depicted in Figure 7 by thin light blue color. In the same figure, we chart their minimum (thick blue) and the minimal distance of QFSK $\delta_{\min }^{2}$ (thick green). The lowest modulation index leading to UMP-QFSK is $\kappa=5 / 6$.

\section{B. Bi-orthonormal modulation is UMP}

According to the results from the preceding section, we see that UMP property does not require an accurate complex-orthonormal alphabet. Inspired also by [10], we have a conjecture that bi-orthonormal modulation is UMP. The Appendix proves the following lemma.

Lemma 15. Bi-orthonormal modulation is UMP.

Remark 16. Interestingly, the symmetrical XC matrix with the same main diagonal is not sufficient in this case, and an extra kind of symmetry, which obeys XOR as well, is required.

\section{UMP-CPM design}

1) CPM basic properties: CPM is a constant envelope modulation (suitable for satellite communication) with more compact spectrum in compare to the linear modulations with constant envelope (with rectangular (REC) modulation pulse). It has a multidimensional alphabet and better spectral properties than FSK (no Dirac pulses in the spectrum and faster asymptotic spectrum attenuation due to the continuous phase). Bandwidth requirements of the considered schemes are investigated in Section VI-C. CPM includes memory [21] and its modulator consists of the discrete part including memory and the non-linear memoryless part [22]. Denominator of CPM modulation index $\kappa$ is proportional to the number of modulator states described by its trellis and the optimal decoder need to perform Viterbi decoding.

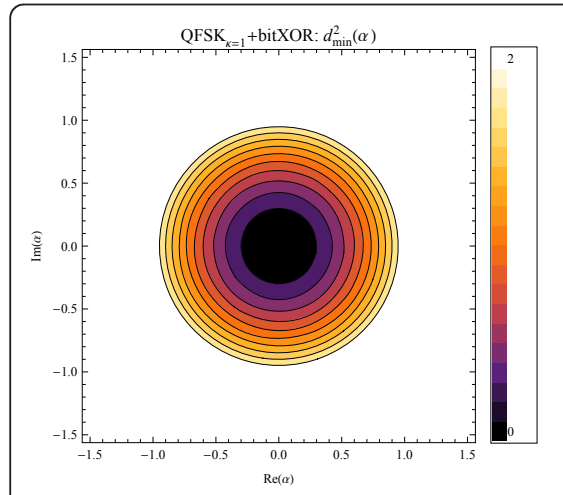

(a) $\kappa=1$

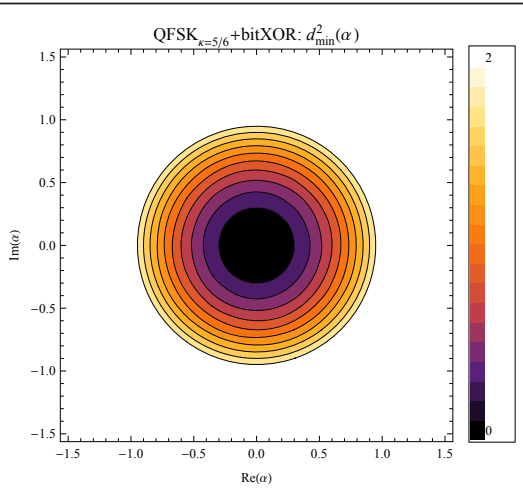

(b) $\kappa=5 / 6$

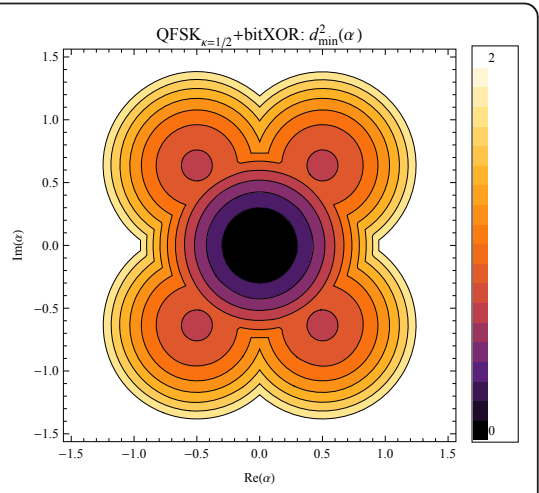

(c) $\kappa=1 / 2$

Figure 5 Parametric minimal distance of uniformly most powerful (UMP) complex-orthonormal QFSK $\kappa=1$ (a) and UMP-QFSK with optimized $\boldsymbol{\kappa}=5 / 6$ (b). QFSK $\boldsymbol{\kappa}=1 / 2$ (c) demonstrates that real-orthogonality does not suffice for UMP property, however its minimal distance is not so poor as in case of QPSK. 


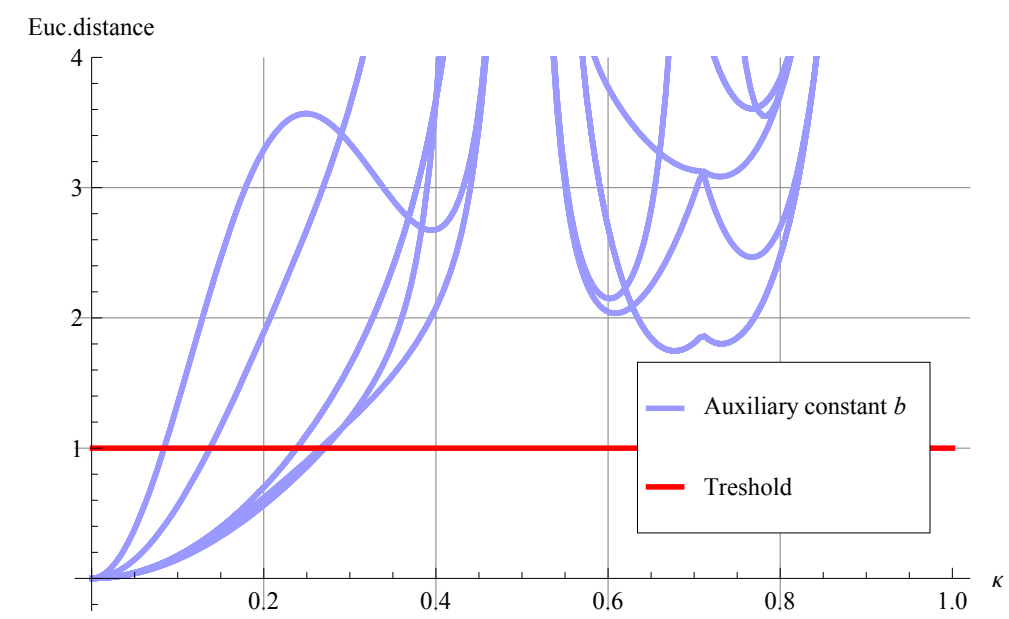

Figure 6 Auxiliary constant $\boldsymbol{b}$ (32) as a function of modulation index $\kappa$ for QFSK. Note, $b$ is always greater than one for practical schemes where $\kappa \geq 1 / 2$.

CPM possess several degrees of freedom; for simplicity, we restrict on the full-response (i.e. the frequency pulse is of the symbol length) and minimum shift $\kappa=1 / 2$ case for which constellation space alphabet is $N_{s}=M_{c}$ dimensional.

2) Design of full-response $\kappa=1 / 2 U M P$-CPM by pulse shape optimization: In the same way, we have excluded channel coding from design of UMP alphabet, we do not need to consider modulation memory of CPM. In our case, the nonlinear memoryless part is determining.

Assumed full-response $\kappa=1 / 2 \mathrm{CPM}$ has the modulation trellis with only two states and the non-linear memoryless alphabet consists of $2 M_{c}$ signals of which the first half starting from the first state have opposite sign than the other half starting from the latter state, see, for example, the trellis of binary scheme in Figure 8 .
Our design is based on Lemma 15, utilizing the above mentioned symmetries, we design a bi-orthonormal UMP modulation simply keeping orthonormal signals starting from the first state.

3) CPM signals notation: Let us denote positive-sign alphabet (signals starting from the zero state) $\mathscr{A}^{+}$and negative-sign alphabet $\mathscr{A}^{-}=-\mathscr{A}^{+}$. The overall alphabet (non-linear memoryless part) is $\mathscr{A}=\left\{\mathscr{A}^{+}, \mathscr{A}^{-}\right\}$. Assuming unit energy signals, full-response $h=1 / 2$ CPM has

$$
\mathscr{A}^{+}=\left\{s_{i}(t)\right\}_{i=0}^{M_{c}-1}=\left\{e^{j \pi\left(\frac{t}{2}\left(M_{c}-1\right)+c \beta(t)\right)}\right\}
$$

where data symbol $c \in\left\{-\left(M_{c}-1\right),-\left(M_{c}-3\right), \ldots,\left(M_{c}-\right.\right.$ $1)\}, t$ is normalized to one symbol duration $t \in[0,1)$ and $\beta(t)$ is a phase pulse.

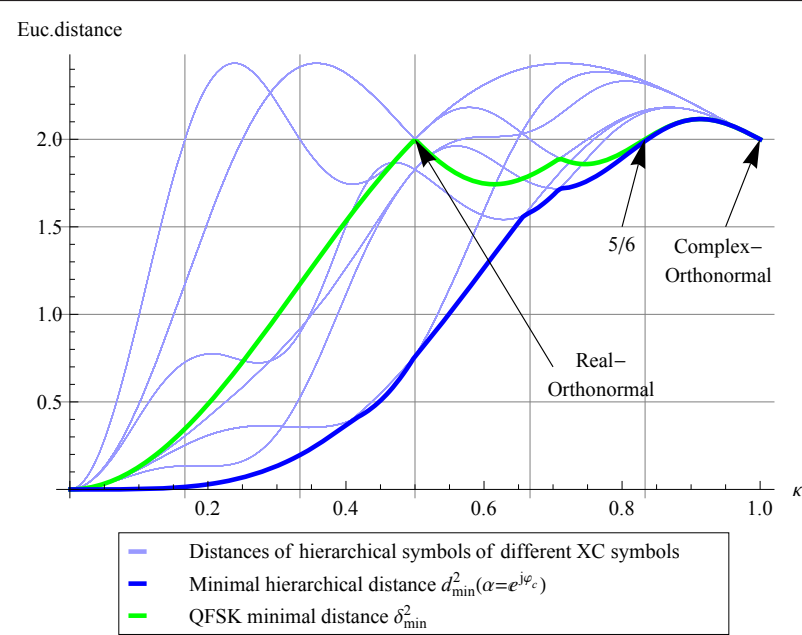

Figure 7 Distances of hierarchical symbols corresponding to different XC symbols for QFSK and the critical parameter value $\alpha=\mathrm{e}^{j \varphi_{c}}$ Minimal value of modulation index fulfilling the UMP condition is $\kappa=5 / 6$ (green thick line meets blue thick line). 


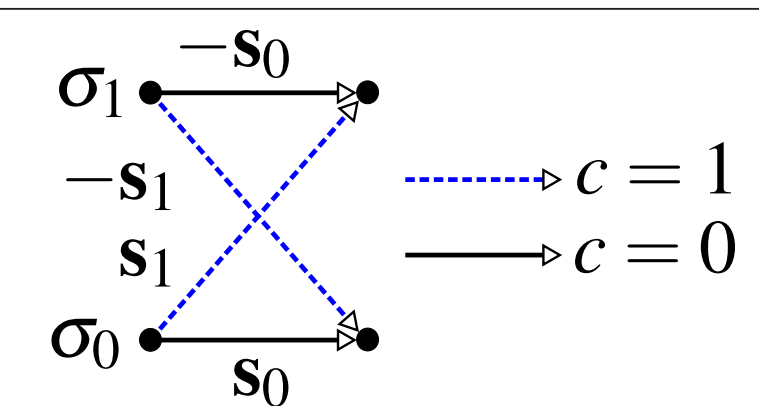

Figure 8 Binary full-response $\boldsymbol{\kappa}=1 / 2$ CPM trellis. Note, if signal space vectors $\mathbf{s}_{0}$ and $\mathbf{s}_{1}$ are orthonormal, than the resulting alphabet is bi-orthonormal.

4) Proposed pulse parametrization: The remaining degree of freedom which we exploit to set the signal correlation is a phase pulse shape. We introduce a simple shaping form obtained as a linear parametrization of Raised Cosine (RC) pulse which we denote as a Scaled $\mathrm{RC}$ (SRC) pulse. The proposed parametric SRC phase pulse is

$$
\beta(t, p)=\frac{1}{2}\left(t-p \frac{\sin 2 \pi t}{2 \pi}\right),
$$

where $p$ is a real parameter. The phase pulse correspond to REC pulse for $p=0$ and to RC pulse for $p=$ 1, see Figure 9.

This parametrization has number of advantages, it does not influence the number of modulator states/signal alphabet cardinality, and it has known analytical formula for bandwidth [23] (roughly the higher $p$ the wider bandwidth).

\section{5) Design of binary UMP-CPM}

Lemma 17. Binary full-response CPM with $\kappa=1 / 2$ and parametric SRC pulse (34) with $p \simeq 2.35$ is UMP.

Proof: Let us consider a binary case, the positive-sign alphabet is

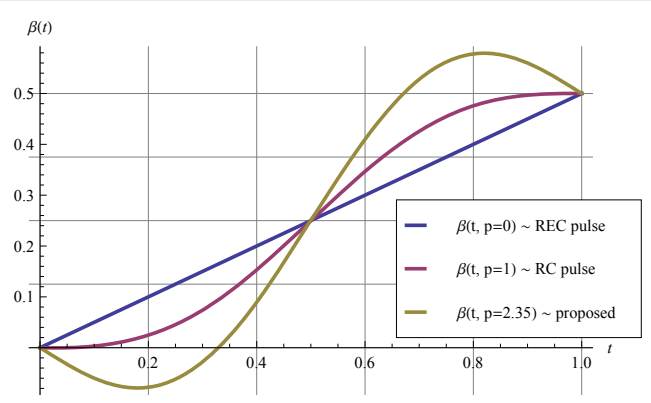

Figure 9 Proposed parametric SRC pulse linearly scale its cosine part.

$$
\mathscr{A}^{+}=\left\{s_{0}(t), s_{1}(t)\right\}=\left\{e^{j \pi\left(\frac{t}{2}+\beta(p, t)\right)}, e^{j \pi\left(\frac{t}{2}-\beta(p, t)\right)}\right\}
$$

The correlation

coefficient $\rho=\left\langle s_{0}(t), s_{1}(t)\right\rangle=\int_{0}^{1} s_{0}(t) s_{1}^{*}(t) \mathrm{d} t$ has an analytic expression in the case of SRC pulse. The expression consists of generalized hyper-geometric functions with a zero real part, see $|\rho|$ in Figure 10.

We conclude that $p \simeq 2.35$ leads to the orthonormal signals, and the lemma is true.

Remark 18. The proposed pulse parametrization has an extra advantage that the squared norm of the signal difference of binary alphabet is always 2 for any $p$. The reason is simply given by zero real part of $\rho$ for any $p$, as has been mentioned in the proof above, then $\| s_{0}(t)$ $s_{1}(t) \|^{2}=2(1-\mathfrak{R}\{\rho\})=2$. Hence, we can adjust the correlation required for UMP condition without affecting the minimal distance $\delta_{\min }^{2}$.

We evaluate parametric minimal distance in Figure 11 to confirm the UMP property of the proposed scheme. We conclude that minimal distance of non-UMP schemes with REC and RC are close to be UMP. In the last section with numerical results, we will see that the error performance of these schemes are practically identical. However, in the case of quaternary/higher-order alphabet, the differences are more significant.

\section{6) Design of quaternary UMP-CPM}

Lemma 19. Quaternary full-response CPM with $\kappa=1 / 2$ and parametric SRC pulse (34) with $p \simeq-7$ or $p \simeq$ 10.2 is UMP.

Proof: The above derivation for binary alphabet can be generalized for any alphabet cardinality; for simplicity, we focus on the quaternary case. Let us consider 4-ary full-response CPM $\kappa=1 / 2$ and SRC pulse; the modulation trellis has the same number of states, see Figure 12.

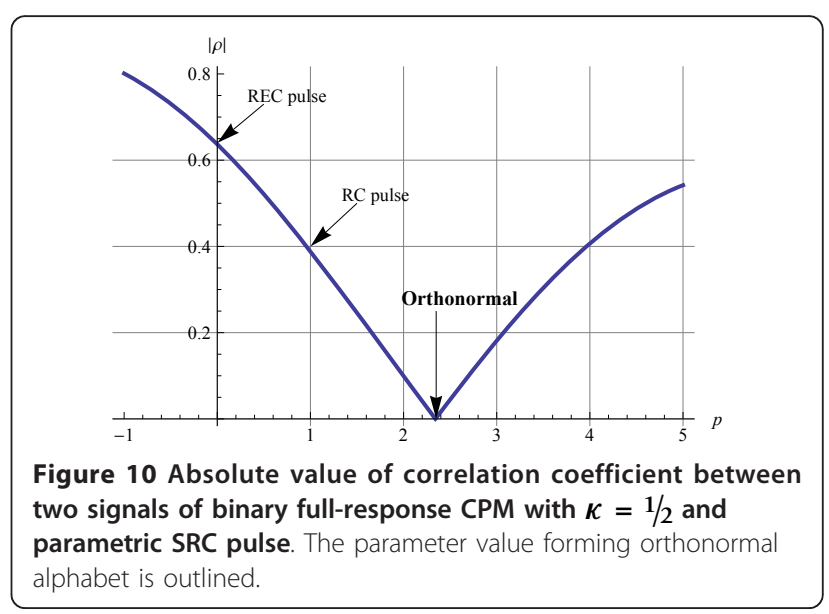




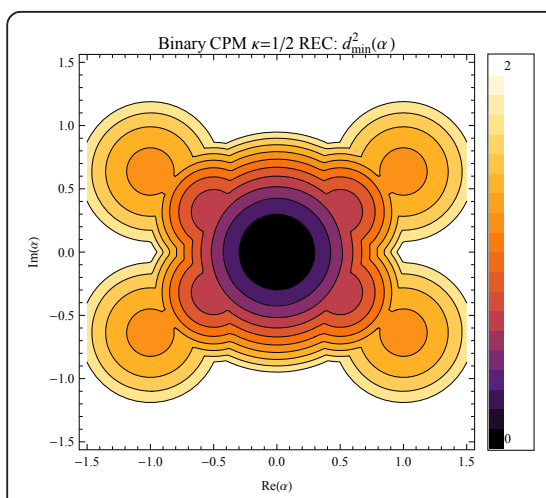

(a) $p=0$

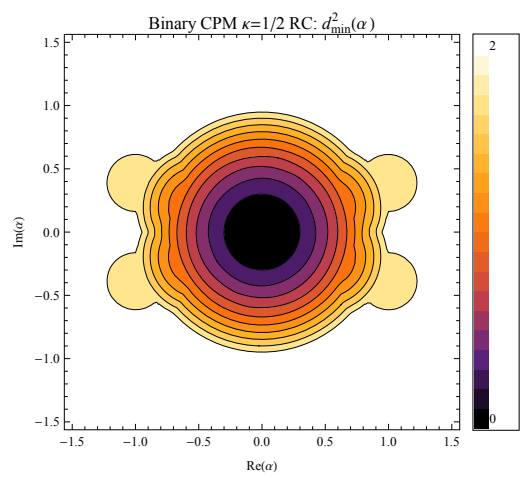

(b) $p=1$

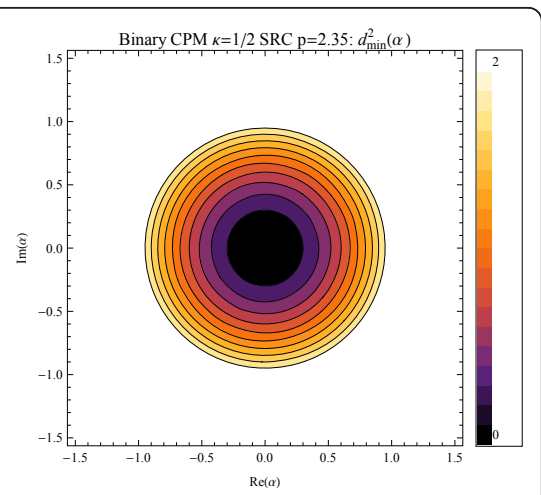

(c) $p \simeq 2.35$

Figure 11 Parametric minimal distance of binary full-response CPM with $\kappa=1 / 2$ and REC pulse (a), RC pulse (b) and UMP SRC pulse with $p \simeq 2.35$ (c).

The positive-sign alphabet is

$$
a^{+}=\left\{e^{j \pi\left(\frac{3}{2} t+c \beta(t, p)\right)}\right\},
$$

where $c \in\{-3,-1,1,3\}$ a data symbol. Our target is by variation of parameter $p$ make set $\mathscr{A}^{+}$orthonormal. There are 4 signals in the set; thus, there are six different signal pairs that must be mutually orthonormal. Let us assume a sum of squared absolute values of individual correlation coefficients (of every signal pairs from $\left.\mathscr{A}^{+}\right) \sum_{i}\left|\rho_{i}\right|^{2}$ as an indication function. This indication function is zero only for orthogonal alphabet. In Figure 13 , we depict the indicating function against parameter $p$, concluding that orthonormal set is obtained, for example, for $p \in\{-7,10.2\}$, which proves the lemma.

To demonstrate the UMP property of the proposed scheme, we evaluate parametric minimal distance with REC, RC and proposed SRC pulse, see Figure 14. Contrary to binary case, the minimal distance of non-UMP

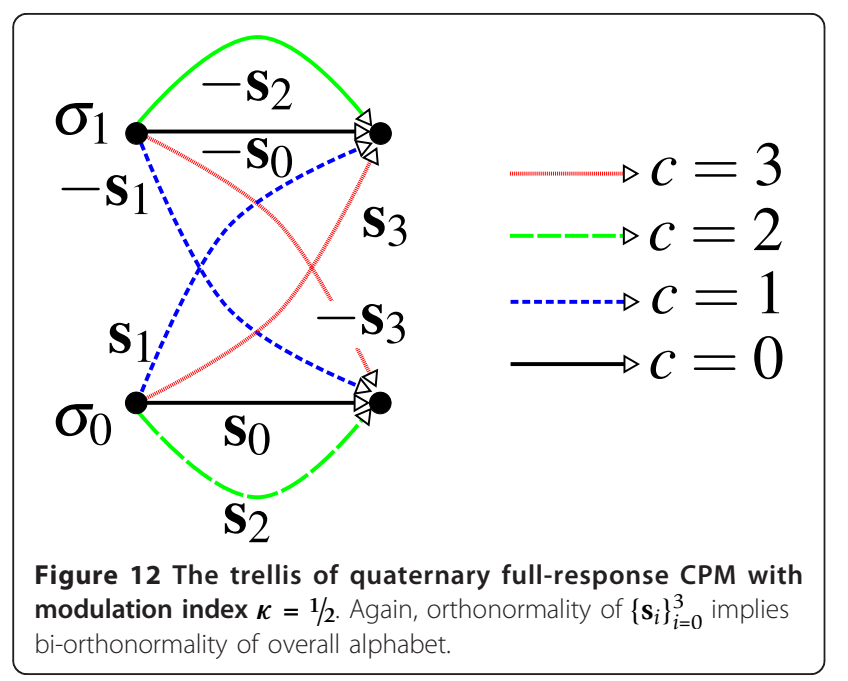

schemes with REC and RC are far to be UMP, and scheme with RC pulse has even lower $\delta_{\min }^{2}=1$, which is in correspondence with the later presented error simulations.

\section{Numerical results}

\section{A. Error performance of memoryless modulations}

In this section, we numerically evaluate symbol error rate (SER) in HDF-MAC stage for several alphabets. We assume simple AWGN channel, frequency-flat uncorrelated Rayleigh/Rice (with Rician factor $K=10 \mathrm{~dB}$ ) fading. As we discussed in Section II-B, we assume uncoded communication and complete channel state information available at the receiving side (CSIR). In Figure 15, we depict following memoryless modulations using XOR XC: nonUMP QPSK, QFSK $\kappa=1 / 2$, UMP-QFSK $\kappa=1$ and proposed UMP-QFSK $\kappa=5 / 6$. All modulation alphabets have the same minimal distance of scalar modulation $\delta_{\min }^{2}$; therefore, the performance in AWGN channel is almost similar. The advantage of UMP alphabets is more evident in fading channels, particularly non-UMP QPSK has

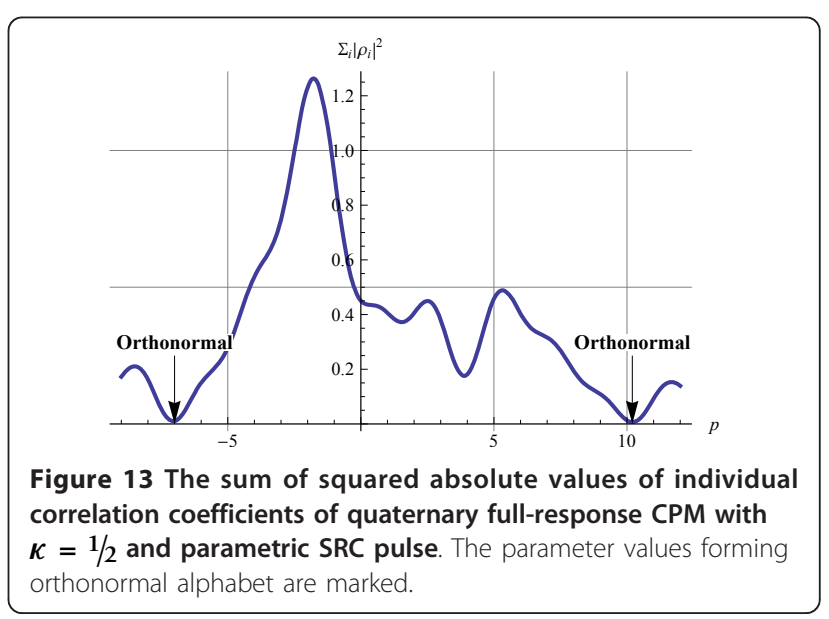




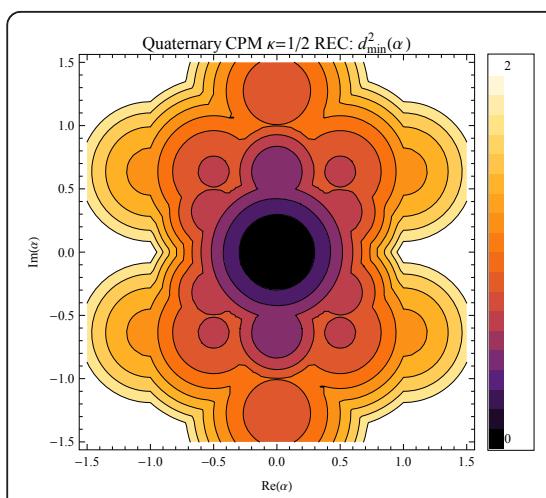

(a) $p=0$

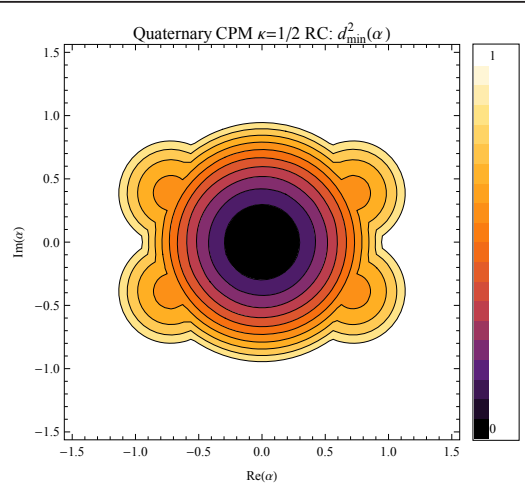

(b) $p=1$

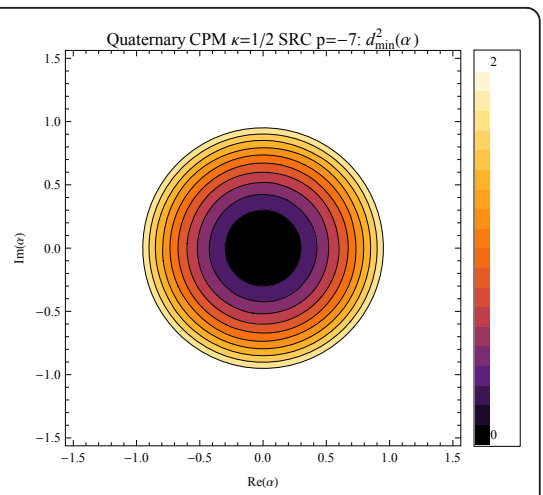

(c) $p \simeq-7$

Figure 14 Parametric minimal distance of quaternary full-response CPM with $\kappa=1 / 2$ and REC pulse (a), RC pulse (b) and UMP SRC pulse with $p \simeq-7$ (c).

roughly $\sim 2 d B$ penalty in Rayleigh fading and even about $\sim 10 d B$ penalty in Rice $K=10 d B$ over UMP alphabets for sufficiently large SNR. It is interesting that non-UMPQFSK $\kappa=1 / 2$ is also well robust to the channel parametrization. Therefore, we may expect that non-linear modulations avoiding all catastrophic parameters are generally more robust to the channel parametrization than the linear one. We supplement the error simulations by related end-to-end throughput simulations including BC stage using for simplicity the same alphabet. We evaluate the throughputs as a relative number of bits of correctly detected 256-bit long packets, see Figure 16.

\section{B. Error performance of full-response CPM}

Here, SER in the MAC stage of non-linear full-response $\mathrm{CPM} \kappa=1 / 2$ with optimized modulation pulses are shown.

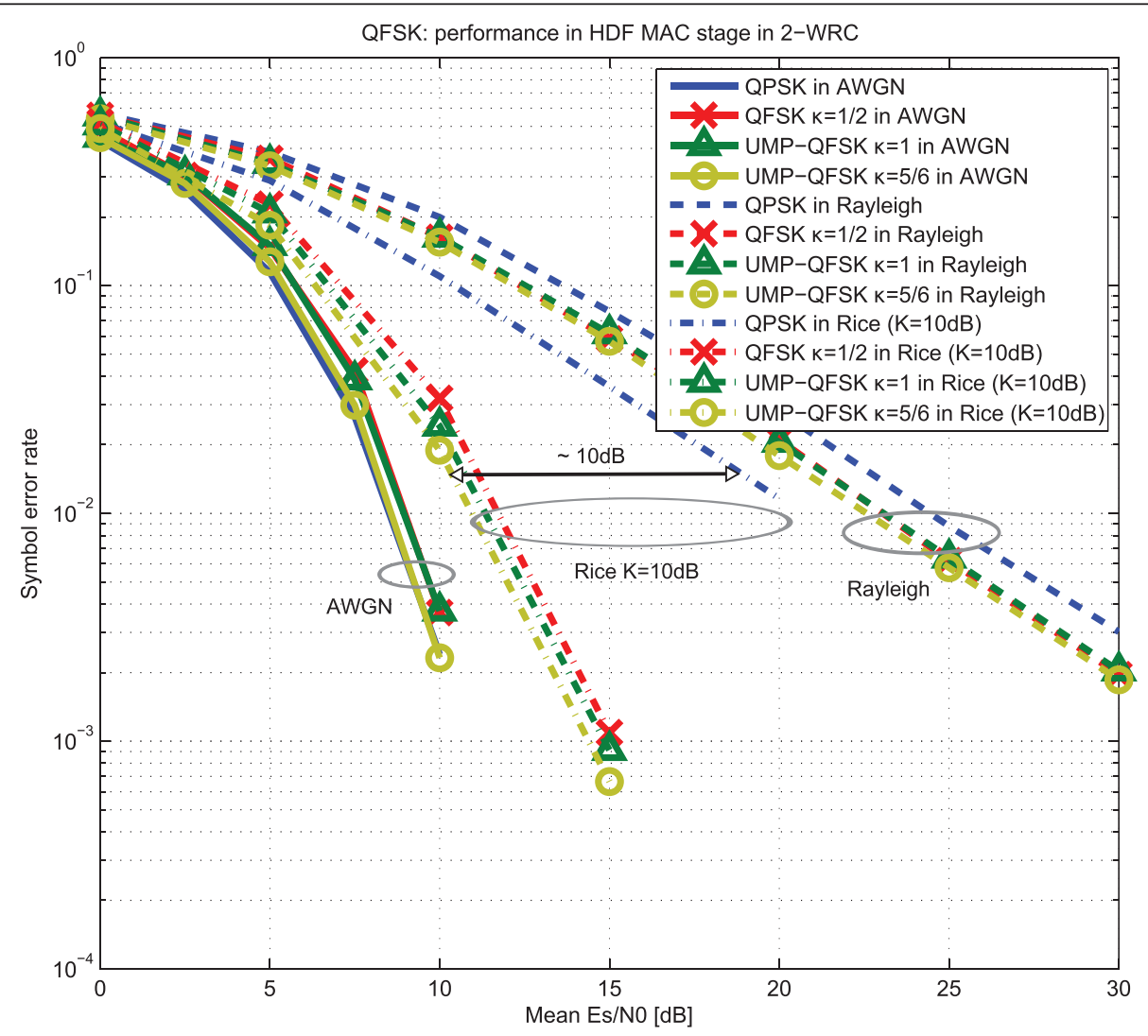

Figure 15 SER in the MAC stage assuming uncoded detection with CSIR in AWGN, Rayleigh and Rice $K=10 \mathrm{~dB}$ fading channel for memoryless modulations: QPSK, QFSK $\kappa=1 / 2$, UMP-QFSK $\kappa=1$ and UMP-QFSK $\kappa=5 / 6$. 


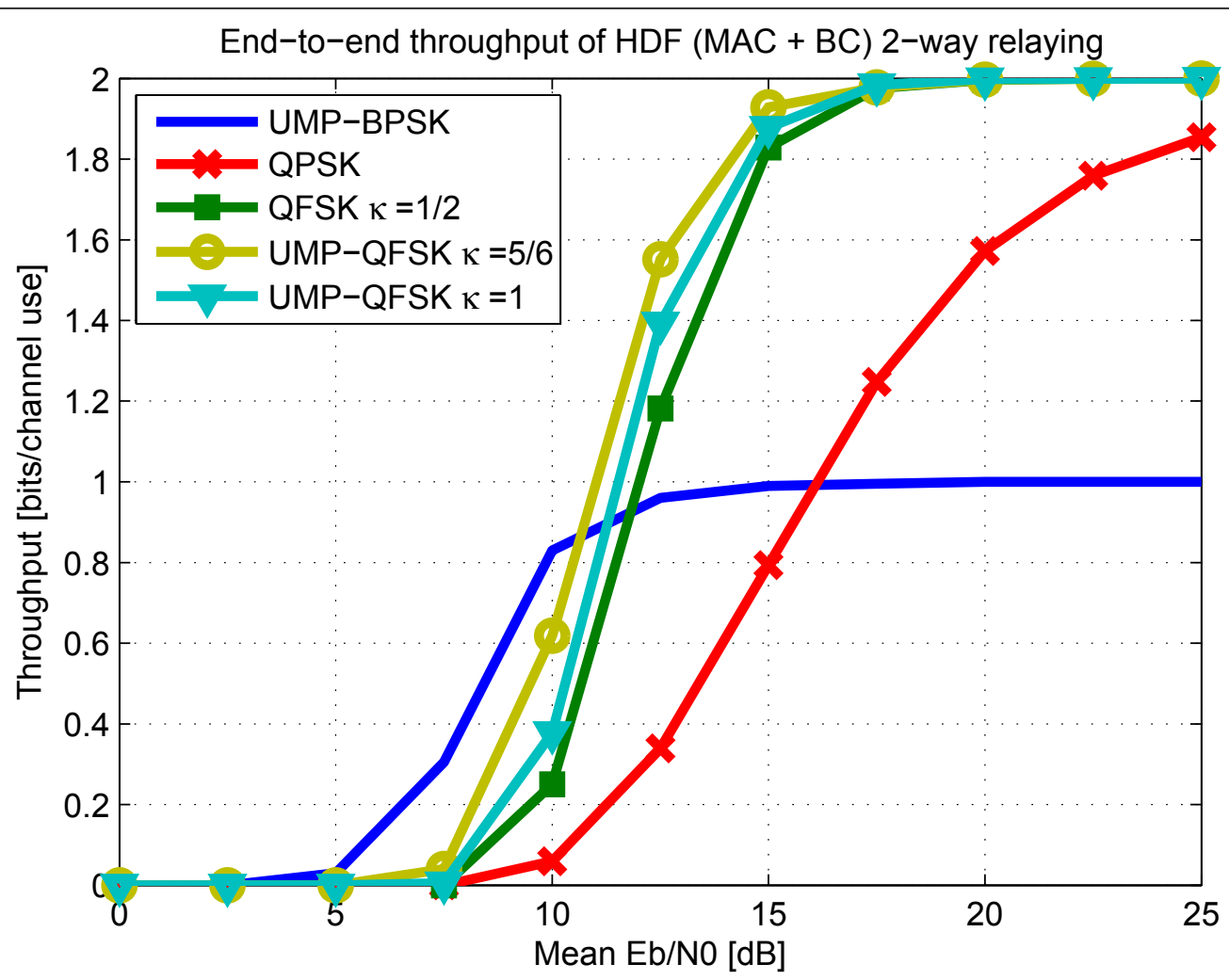

Figure 16 End-to-end throughput of HDF strategy (including MAC and BC stage) assuming uncoded detection with CSIR in Rice $K=$ $10 \mathrm{~dB}$ fading channel for memoryless modulations: UMP-BPSK, QPSK, QFSK $\kappa=1 / 2$, UMP-QFSK $\kappa=1$ and UMP-QFSK $\kappa=5 / 6$.

We have seen that the presence of discrete memory does not influence the UMP property, although it cannot be ignored at the receiver side. We use a joint $\left[\partial_{A}, \partial_{B}\right]$ decoding algorithm based on the vector Viterbi algorithm [24] describing the structure of receiving signals by a supertrellis with super-states. Each super-state is a vector of states that join together the actual state at the node $\mathrm{A}$ with the state at the node $\mathrm{B}$. Then, the joint estimate of $\left[\partial_{A}, \partial_{B}\right]$ is obtained by the sequence Viterbi algorithm over the super-trellis. Thereafter, the exclusively coded data symbols are obtained as $\partial_{A B}=\partial_{A} \oplus \partial_{B}$.

In Figure 17, it is depicted SER of binary full-response CPM $\kappa=1 / 2$ with REC, RC and proposed SRC pulse and also UMP-BPSK modulation as a reference. We describe binary full-response CPM $\kappa=1 / 2$ as a minimum shift keying (MSK) modulation to shorten the notation, though MSK strictly use the REC pulse. We conclude that non-UMP schemes with different pulses have almost the same performance, as we expected from Figure 11. Therefore, choosing a pulse with the narrowest spectra (REC) is appropriate. In this case, the proposed SRC pulse has only theoretical value as a performance benchmark.

The proposed SRC pulse is more advantageous for quaternary alphabet, see Figure 18 . We shortly denote quaternary full-response CPM $\kappa=1 / 2$ as a QMSK modulation. We observe that performance with REC pulse is close to the UMP alphabet performance, but contraintuitively the performance with RC pulse is by several $\mathrm{dBs}$ worse even in the AWGN channel. The REC pulse, in this case, is a practical choice because the proposed SRC pulse requires more bandwidth, see the bandwidth comparison in the following section.

\section{Bandwidth comparison}

Bandwidth requirement of the considered modulations is presented in Table 2. The first part of the table describes bandwidth of linear memoryless alphabets (denoted shortly as 'Linear $\mathscr{A}$ ') which is solely given by used pulse function [20]. We present well-known rootraised cosine (RRC) pulse parametrized by roll-off factor $\lambda$ whose compact and finite bandwidth is $W=(1+\lambda) / 2 T_{s}$. Due to the finiteness of the bandwidth, ideal RRC has infinite time duration. Vice versa, rectangular (REC) pulse finite in temporal domain has infinite bandwidth and in this case, we use the fractional power-containment bandwidth definition where $W_{99 \%}$ is a bandwidth containing $99 \%$ of the total signal power. In this paper, all linear modulations (with one complex dimension including BPSK) use the same bandwidth. Note the 


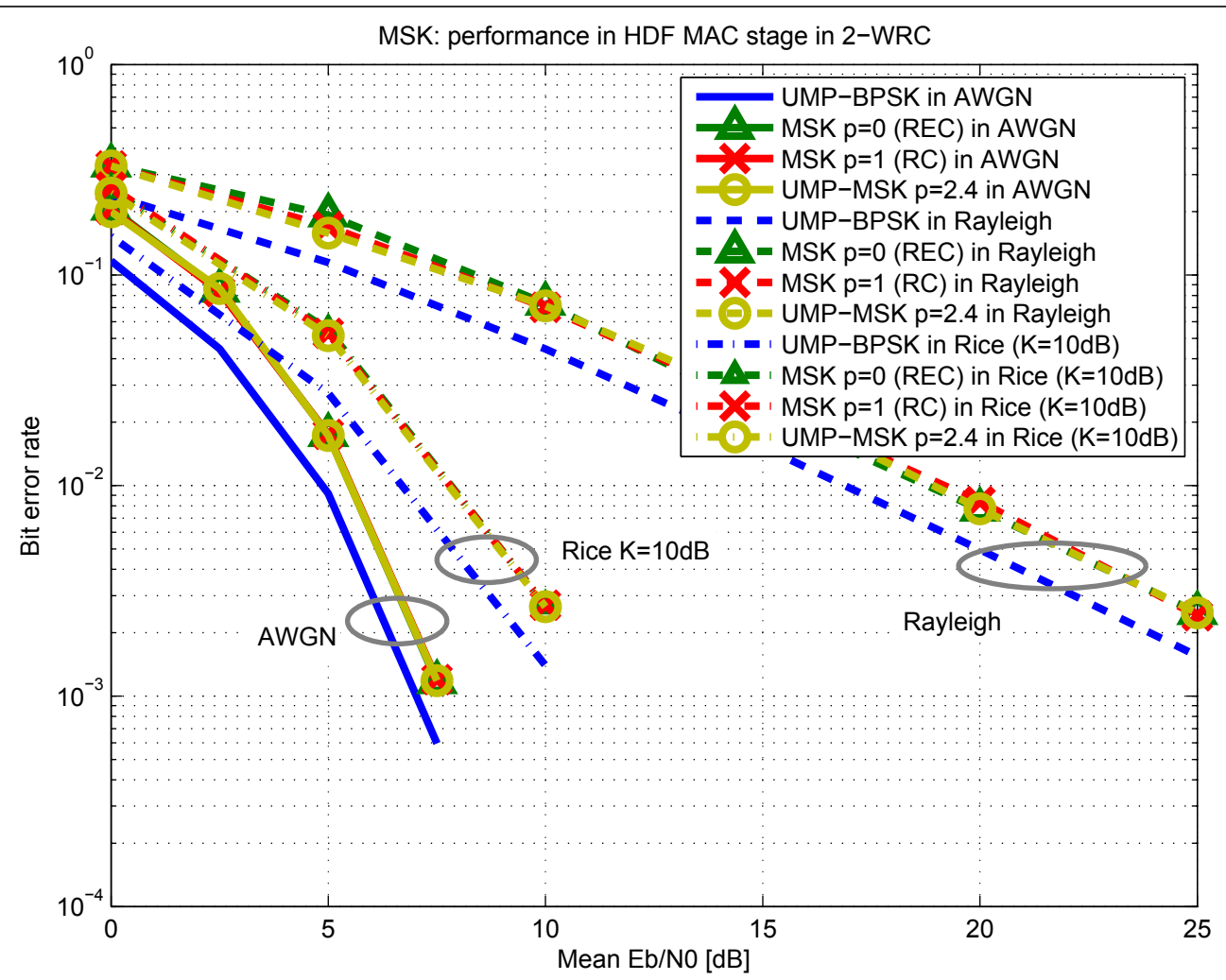

Figure 17 SER in the MAC stage assuming uncoded detection with CSIR in AWGN, Rayleigh and Rice $K=10 \mathrm{~dB}$ fading channel of binary full-response CPM $\kappa=1 / 2$ (MSK) with REC, RC and proposed SRC pulse. Additionally $w$ depict UMP-BPSK as a reference.

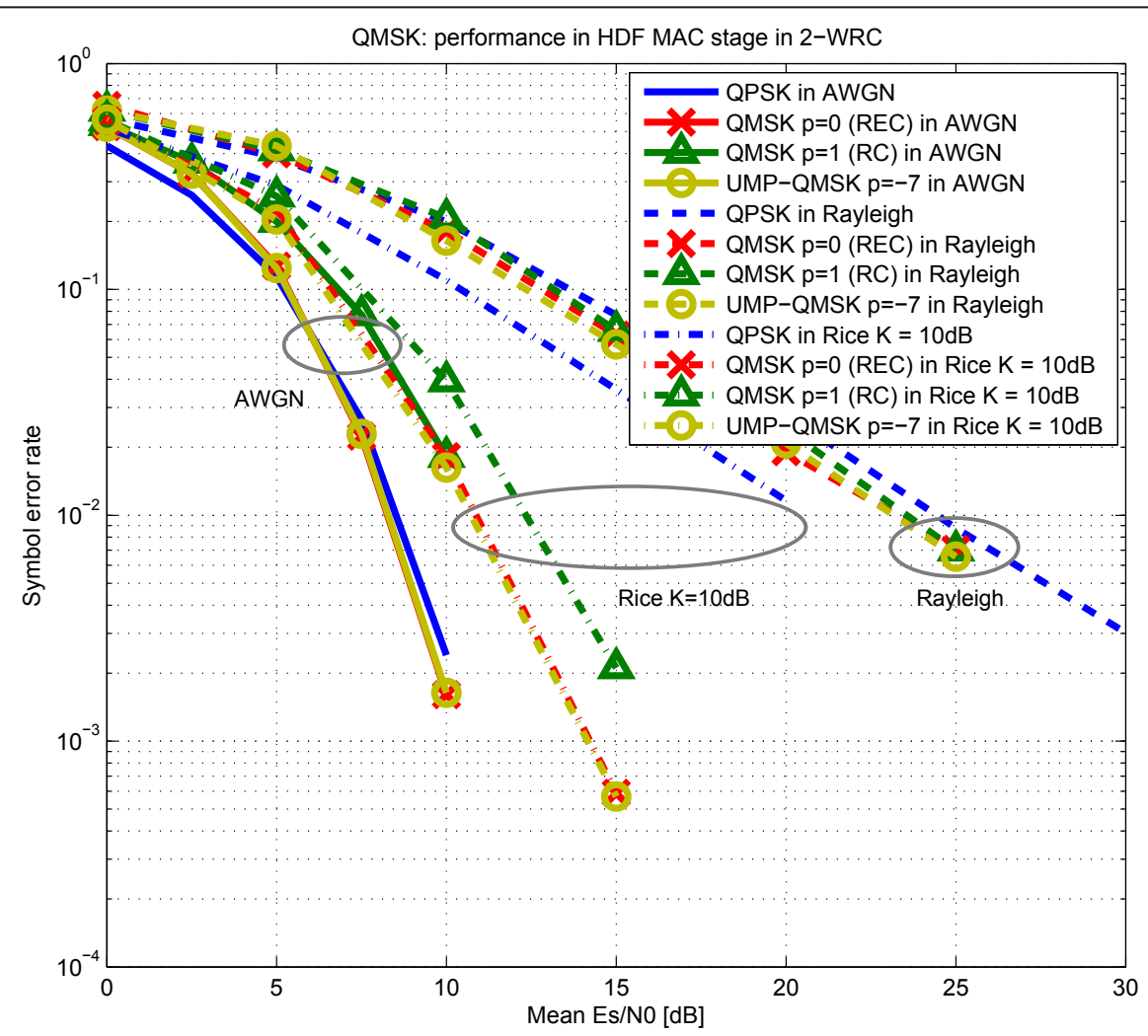

Figure 18 SER in the MAC stage assuming uncoded detection with CSIR in AWGN, Rayleigh and Rice $K=10 \mathrm{~dB}$ fading channel of quaternary full-response CPM $\kappa=1 / 2$ (QMSK) with REC, RC and proposed SRC pulse. Additionally, we depict non-UMP QPSK as a 
Table 2 Bandwidth comparison

\begin{tabular}{cccc}
\hline$\nabla$ Linear $\mathscr{A}$ & RRC $\boldsymbol{\lambda}=\mathbf{0}$ & RRC $\boldsymbol{\lambda}=\mathbf{1}$ & REC \\
\hline$W_{5}$ & 0.5 & 1 & 10.3 \\
$\nabla$ QFSK & $\kappa=1 / 2$ & $\kappa=5 / 6$ & $\kappa=1$ \\
$W_{99 \%} T_{s}$ & 10.9 & 15 & 21 \\
$\diamond \mathrm{MSK}$ & $p=0$ & $p=1$ & $p=2.35$ \\
$W_{99 \%} T_{s}$ & 0.6 & 1.1 & 1.5 \\
$\diamond \mathrm{QMSK}$ & $p=0$ & $p=1$ & $p=-7$ \\
$W_{99 \%} T_{s}$ & 1.4 & 1.8 & 5.9 \\
\hline
\end{tabular}

significant difference of error performance between BPSK and QPSK in Figure 15 and 17. BPSK is UMP only if it uses a single entire complex dimension (entire bandwidth); thus, it needs one real dimension more than in point-to-point communication to be UMP.

The other part of the table is dedicated to non-linear modulations. We denote full-response CPM $\kappa=1 / 2$ with SRC pulse by acronym 'MSK'. Its fractional bandwidth, analytically described in [23], shows that bi-orthogonal case require more bandwidth than e.g. the case with $p=$ 0 . The similar trend has also bandwidth of QMSK which we obtained according to [21], see average power spectral densities in Figure 19.

\section{Conclusion and discussions}

In this paper, we have investigated modulations and exclusive codes (XC; network codes-like) robust to the parametrization for hierarchical-decode-and-forward (HDF) with channel state information at the receiver (CSIR) not requiring any channel adaptation techniques. We have found that such modulation is BPSK and definitely not the other linear modulations (PSK, QAM,...) with higher cardinality than 2 , because they possess socalled catastrophic parameters. The catastrophic parameter is such a non-zero channel parameter that causes zero hierarchical minimal distance strongly degrading overall average performance. It is interesting that minimal cardinality XC (Latin square) should have symmetrical XC matrix with the same diagonal not to imply catastrophic parameters. These conditions fulfills bit-wise $\mathrm{XOR}$, which is the unique solution for binary and quaternary alphabet. It has been shown that to avoid the catastrophic parameters, modulations with more than a single complex dimension have to be considered. This inspired us to assume non-linear frequency modulations naturally having multidimensional waveforming alphabet. We have precisely defined uniformly most powerful (UMP) alphabets, which not only obviate all catastrophic parameters but also reach the minimal distance upper bound for all parameter values. UMP can be interpreted as the most suitable type of unavoidable channel parametrization, and among all alphabets with identical minimal distance of the single alphabet, the UMP alphabets have the best performance serving as a performance benchmark. It is proved that any binary, non-binary complex-

\section{Average Power Spectrum Density}

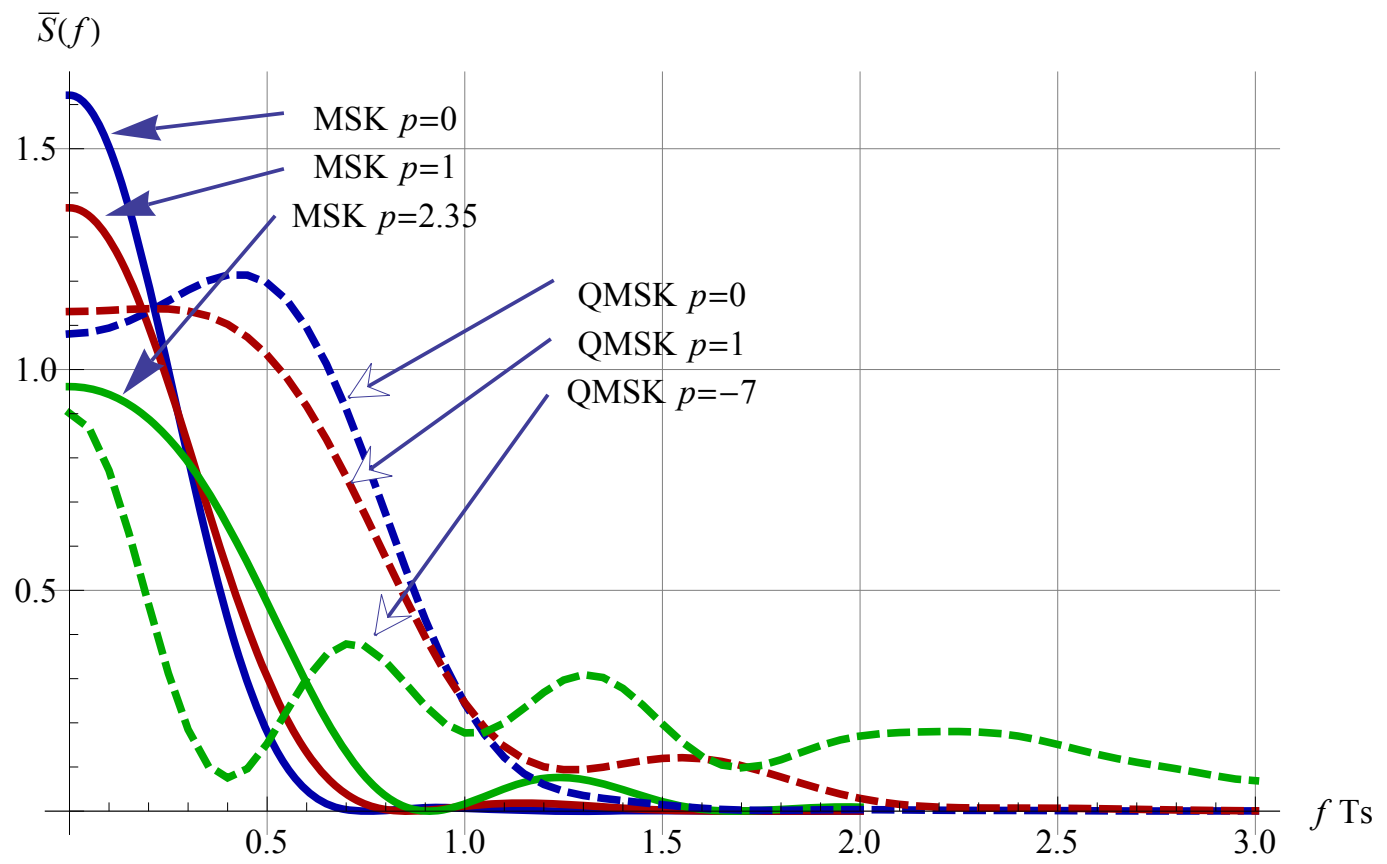

Figure 19 Average power spectral densities of binary and quaternary full-response CPM $\kappa=1 / 2$ (MSK and QMSK) for several different values of parameter $p$. 
orthogonal and non-binary complex-bi-orthogonal alphabets are UMP.

We have found that the considered frequency shift keying (FSK) and full-response $\kappa=1 / 2$ continuous phase modulation (CPM) avoid catastrophic parameters, and we have optimized their parameters (modulation index for FSK and phase pulse shape for CPM) to meet UMP. Based on the numerical simulations, we conclude that existence of catastrophic parameters is much more detrimental to the average performance than violation of UMP condition. The proposed alphabets are summarized in Table 3; the note 'close to UMP' means that despite the alphabet is not UMP, it performs very close to the UMP benchmark.

We have analyzed error and bandwidth performance of the used modulations in Section VI. However, the optimal modulation choice will depend, besides error-bandwidth performance, on the other properties such as complexity or hardware requirements. For instance, BPSK with RRC pulse and MSK $p=0$ have comparable error-bandwidth performance, but MSK possess constant envelope (which allows more efficient power amplifier) at the price of more complex decoding processing (includes e.g. Viterbi algorithm). If we insist on constant envelope feature than MSK $p=0$ needs to be compared with BPSK with REC which requires much more bandwidth. BPSK with REC pulse and two times shorter pulse duration (to deliver 2 bits per channel use) have similar error performance but two times wider bandwidth than QFSK $\kappa=1 / 2$. On the other hand, QFSK receiver consists of parallel bank of matched filters (number of filters equals to the dimensionality) while BPSK receiver has only one filter. QMSK $p=0$ is more preferable than QFSK $\kappa=1 / 2$ since it owns narrower bandwidth, if we can afford slightly more complex decoding processing (e.g., Viterbi algorithm).

\section{Appendix}

Proof (Bi-orthonormal modulation is UMP): Let us consider the modified condition (28) which we have used in UMP-FSK derivation. It states that UMP alphabet modulation fulfills

$$
\|\left|\mathbf{s}_{c_{A}}-\mathbf{s}_{c_{A^{\prime}}{ }^{\prime}}\right|^{2}+|\alpha|^{2}|| \mathbf{s}_{c_{B}}-\left.\mathbf{s}_{c_{B}^{\prime}}\right|^{2}-2|\alpha||| \mathbf{s}_{c_{A}}-\mathbf{s}_{c_{A^{\prime}}^{\prime}}, \mathbf{s}_{c_{B}}-\mathbf{s}_{c_{B^{\prime}}}|| \geq|\alpha|^{2} \delta_{\min }^{2}
$$

for $c_{A} \neq c_{A}{ }^{\prime}, c_{B} \neq c_{B}{ }^{\prime}, c_{A} \oplus c_{B} \neq c_{A}{ }^{\prime} \oplus c_{B}{ }^{\prime}$, where $c_{A}, c_{B}, c_{A}{ }^{\prime}, c_{B}{ }^{\prime}, \in \mathbb{Z}_{M_{c}}$ and $\forall \alpha \in \mathbb{C},|\alpha| \leq 1$. Assume a biorthonormal modulation for which two bi-orthonormal signals have one of the three possible mutual relations. They are orthonormal (described by symbol $\perp$ ), or the same $\leftleftarrows$ or have the opposite sign $\leftrightarrows$. According to the mutual relation, its inner product and squared norm of its difference is

$$
\left\langle\mathbf{s}_{c_{A^{\prime}}}, \mathbf{s}_{c_{B}}\right\rangle=\left\{\begin{array}{rl}
0, & \perp \\
1, & \leftleftarrows \\
-1, & \leftrightarrows
\end{array} \quad\left\|\mathbf{s}_{c_{A}}-\mathbf{s}_{c_{B}}\right\|^{2}=\left\{\begin{array}{l}
2, \perp \\
0, \leftleftarrows . \\
4, \leftrightarrows
\end{array}\right.\right.
$$

In the first step, we analyze all possible values of the inner product term

$$
\begin{aligned}
& Z=\left|\left\langle\mathbf{s}_{C_{A}}-\mathbf{s}_{C_{A^{\prime}},} \mathbf{s}_{C_{B}}-\mathbf{s}_{C_{B}{ }^{\prime}}\right\rangle\right| \\
& =\left|\left\langle\mathbf{s}_{c_{A^{\prime}}} \mathbf{s}_{c_{B}}\right\rangle+\left\langle\mathbf{s}_{c_{A^{\prime}},} \mathbf{s}_{C_{B}{ }^{\prime}}\right\rangle-\left\langle\mathbf{s}_{c_{A^{\prime}}} \mathbf{s}_{c_{B^{\prime}}}\right\rangle-\left\langle\mathbf{s}_{c_{A^{\prime}},} \mathbf{s}_{c_{B}}\right\rangle\right| .
\end{aligned}
$$

Without taking into account the restrictions $c_{A} \neq c_{A}$, $c_{B} \neq c_{B}{ }^{\prime}$ and $c_{A} \oplus c_{B} \neq c_{A}{ }^{\prime} \oplus c_{B}{ }^{\prime}$, the term $z \in\{0,1,2$, $3,4\}$. Note, if the term $z=0$, then the inequality (37) is fulfilled because by definition $\left\|\mathbf{s}_{C_{B}}-\mathbf{s}_{c_{B}}\right\|^{2} \geq \delta_{\min }^{2}$. There are four inner products in (39) that can take three different geometrical arrangements $(\perp, \leftleftarrows, \leftrightarrows)$ altogether there is $3^{4}=81$ combinations. We will show by exhaustive listing of all cases where $z \neq 0$ that such a case

a) cannot happen because the inner products give such results that geometrically this situation cannot exist (e.g. two vectors cannot be orthonormal and perpendicular at the same time) or

b) the inequality (37) is still fulfilled or c) this situation is excluded by XOR symmetries concluding that always $z=0$ which proves the lemma.

In the second step, we show that $z \neq 1$ because always the case a) happen. Due to the space limitation, we will not list all that cases; in fact, considering geometrical properties, it is sufficient to list only those schemes which have different geometrical arrangement. Let us denote the geometrical arrangement by a vector of inner products. The ordered configurations leading to $z=1$ are

Table 3 Summary of the proposed alphabets

\begin{tabular}{ccc}
\hline Alphabet & UMP relation & Notes \\
\hline BPSK & UMP & optimal binary alphabet \\
QFSK $\kappa=1 / 2$ & close to UMP & not optimal spectra \\
QFSK $\kappa=5 / 6$ & UMP & more bandwidth than QFSK $\kappa=1 / 2$ \\
MSK $p=0$ & close to UMP & better spectrum than FSK; include memory \\
QMSK $p=0$ & close to UMP & better spectrum than FSK; include memory \\
MSK $p \simeq 2.35$ & UMP & more bandwidth than MSK $p=0$ \\
QMSK $p \simeq-7$ & UMP & more bandwidth than QMSK $p=0$ \\
\hline
\end{tabular}




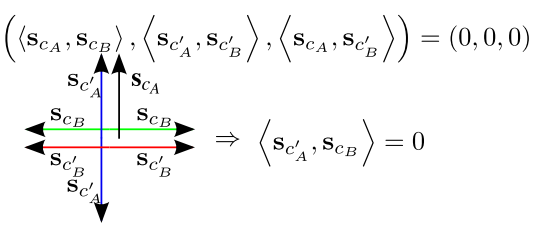

Figure 20 Possible geometrical configurations $\left(\left\langle\mathbf{s}_{c_{A}}, \mathbf{s}_{c_{B}}\right\rangle,\left\langle\mathbf{s}_{c_{A^{\prime}},}, \mathbf{s}_{c_{B^{\prime}}}\right\rangle,\left\langle\mathbf{s}_{c_{A^{\prime}}}, \mathbf{s}_{c_{B^{\prime}}}\right\rangle\right)=(0,0,0)$ imply $\left\langle\mathbf{s}_{c_{A^{\prime}}}, \mathbf{s}_{c_{B}}\right\rangle=\mathbf{0}$

$$
\kappa=1 / 2
$$

For example, let us assume the situation where $\left(\left\langle\mathbf{s}_{C_{A}}, \mathbf{s}_{C_{B}}\right\rangle,\left\langle\mathbf{s}_{C_{A^{\prime}}}, \mathbf{s}_{C_{B}{ }^{\prime}}\right\rangle,\left\langle\mathbf{s}_{c_{A^{\prime}}} \mathbf{s}_{C_{B}{ }^{\prime}}\right\rangle,\left\langle\mathbf{s}_{C_{A^{\prime}},}, \mathbf{s}_{c_{B}}\right\rangle\right)=(0,0,0,1)$. In Figure 20, we draw all possible configurations corresponding to the first three inner products $\left(\left\langle\mathbf{s}_{c_{A}}, \mathbf{s}_{C_{B}}\right\rangle\left\langle\mathbf{s}_{c_{A^{\prime}},}, \mathbf{s}_{{C_{B}}^{\prime}}\right\rangle\left\langle\mathbf{s}_{c_{A^{\prime}}}, \mathbf{s}_{c_{B}{ }^{\prime}}\right\rangle\right)=(0,0,0)$, by that we demonstrate that it implies the last term to be $\left\langle\mathbf{s}_{c_{A^{\prime}}}, \mathbf{s}_{c_{B}}\right\rangle=0$. In this case, it is not true; hence, this example situation would never occur. Hereby, the situations with any permutation of the inner products $(0,0$, $1,0),(0,1,0,0)$, etc. are also excluded. In Figure 21 and 22 ,

we similarly show that configuration $(1,1,1) \Rightarrow 1$ and $(-1,-1,-1) \Rightarrow-1$. We denote these three situations above as a $(x, x, x) \Rightarrow x$ law. The last remaining configurations of $z=1,(40)$, which are not excluded by the $(x, x, x) \Rightarrow$ $x$ law, are $(0,1,1,-1)$ and $(0,1,-1,-1)$. They are again excluded by a), see Figures 23 and 24 . Since we excluded all situations where $z=1$ then $z \neq 1$.

In the last third step, we will conclude that $|\alpha|=1$ is critical and check all the remaining situations/configurations where $z \in\{2,3,4\}$. We will see that either a), b)

$$
\begin{gathered}
\left(\left\langle\mathbf{s}_{c_{A}}, \mathbf{s}_{c_{B}}\right\rangle,\left\langle\mathbf{s}_{c_{A}^{\prime}}, \mathbf{s}_{c_{B}^{\prime}}\right\rangle,\left\langle\mathbf{s}_{c_{A}}, \mathbf{s}_{c_{B}^{\prime}}\right\rangle\right)=(1,1,1) \\
\mathbf{s}_{c_{B}^{\prime}}^{\prime} \mathbf{s}_{c_{B}} \mathbf{s}_{c_{A}^{\prime}} \mathbf{s}_{c_{A}} \\
\uparrow
\end{gathered}
$$

Figure 21 Possible geometrical configurations $\left(\left\langle\mathbf{s}_{c_{A}}, \mathbf{s}_{c_{B}}\right\rangle,\left\langle\mathbf{s}_{c_{A^{\prime}},}, \mathbf{s}_{c_{B_{B}^{\prime}}}\right\rangle,\left\langle\mathbf{s}_{c_{A}}, \mathbf{s}_{c_{B^{\prime}}}\right\rangle\right)=(1,1,1)$ imply $\left\langle\mathbf{s}_{c_{A^{\prime}}}, \mathbf{s}_{c_{B}}\right\rangle=\mathbf{1}$

$$
\begin{aligned}
& \left(\left\langle\mathbf{s}_{c_{A}}, \mathbf{s}_{c_{B}}\right\rangle,\left\langle\mathbf{s}_{c_{A}^{\prime}}, \mathbf{s}_{c_{B}^{\prime}}\right\rangle,\left\langle\mathbf{s}_{c_{A}}, \mathbf{s}_{c_{B}^{\prime}}\right\rangle\right)=(-1,-1,-1) \\
& \left.\mathbf{s}_{c_{B}^{\prime}} \mathbf{s}_{c_{A}^{\prime}} \uparrow\right|_{\boldsymbol{A}^{\prime}} \mathbf{s}_{c_{A}} \Rightarrow\left\langle\mathbf{s}_{c_{A}^{\prime}}, \mathbf{s}_{c_{B}}\right\rangle=-1
\end{aligned}
$$

Figure 22 Possible geometrical configurations $\left(\left\langle\mathbf{s}_{c_{A^{\prime}}}, \mathbf{s}_{c_{B}}\right\rangle,\left\langle\mathbf{s}_{c_{A^{\prime}},}, \mathbf{s}_{c_{B^{\prime}}}\right\rangle,\left\langle\mathbf{s}_{c_{A^{\prime}}}, \mathbf{s}_{c_{B}{ }^{\prime}}\right\rangle\right)=(-1,-1,-1)$ imply $\left\langle\mathbf{s}_{c_{A}{ }^{\prime}}, \mathbf{s}_{c_{B}}\right\rangle=-1$

$$
\begin{array}{r}
\left\langle\left\langle\mathbf{s}_{c_{A}}, \mathbf{s}_{c_{B}}\right\rangle,\left\langle\mathbf{s}_{c_{A}^{\prime}}, \mathbf{s}_{c_{B}^{\prime}}\right\rangle,\left\langle\mathbf{s}_{c_{A}}, \mathbf{s}_{c_{B}^{\prime}}\right\rangle\right)=(1,1,-1) \\
\mathbf{s}_{c_{B}} \\
\left.\mathbf{s}_{c_{B}^{\prime}}\right\rceil_{\downarrow \downarrow} \mathbf{s}_{c_{A}} \\
\Rightarrow\left\langle\mathbf{s}_{c_{A}^{\prime}}, \mathbf{s}_{c_{B}}\right\rangle=-1 \\
\mathbf{s}_{c_{A}^{\prime}}
\end{array}
$$

Figure 23 Possible geometrical configurations $\left(\left\langle\mathbf{s}_{c_{A^{\prime}}}, \mathbf{s}_{c_{B}}\right\rangle,\left\langle\mathbf{s}_{c_{A^{\prime}},} \mathbf{s}_{c_{B^{\prime}}}\right\rangle,\left\langle\mathbf{s}_{c_{A^{\prime}}}, \mathbf{s}_{c_{B}{ }^{\prime}}\right\rangle\right)=(1,1,-1)$ imply $\left\langle\mathbf{s}_{c_{A^{\prime}}}, \mathbf{s}_{c_{B}}\right\rangle=-1$

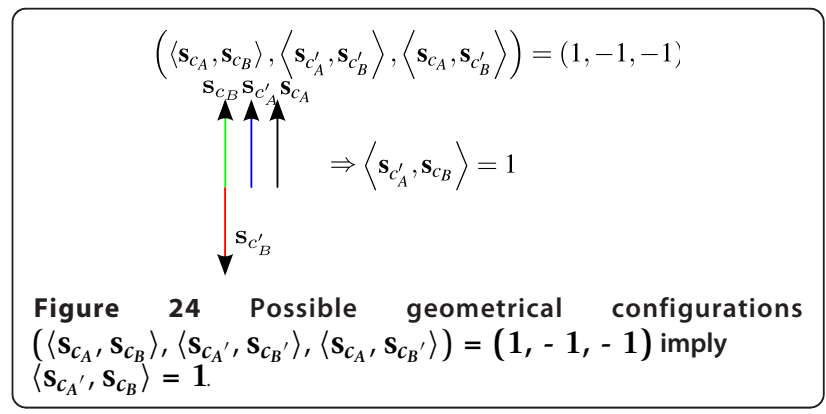

or c) happen and thus always $z=0$. We have seen in the Section V-A that $|\alpha|=1$. is critical if the term $b=\left|\left\langle\mathbf{s}_{c_{A}}-\mathbf{s}_{c_{A^{\prime}}{ }^{\prime}} \mathbf{s}_{c_{B}}-\mathbf{s}_{c_{B}}\right\rangle\right| /\left\|\mathbf{s}_{c_{B}}-\mathbf{s}_{c_{B^{\prime}}}\right\|^{2}-\delta_{\text {min }}^{2}$ is $b \geq 1$. In our case, $b=z / 2$ and assuming $z \in\{2,3,4\}$ always $b \geq$ 1 and so a critical parameter is $|\alpha|=1$.

The configurations corresponding to the case $z=2$ are

$(0,0,1,1),(0,0,-1,-1),(0,1,-1,0),(1,1,1,-141)$

The configuration $(0,0,1,1)$ entails that $\mathbf{s}_{c_{A}}=\mathbf{s}_{C_{B}}$ $\& \mathbf{s}_{c_{A}{ }^{\prime}}=\mathbf{s}_{C_{B}}$ thus implies $k=n \& l=m$ and is excluded by $\mathrm{XC}$ with symmetrical XC matrix which we already assume. The configuration $(0,0,-1,-1)$ is rather problematic, see its configuration in Figure 25.

Without deep analysis, we conclude that whatever mapping between waveforms $\mathbf{s}_{c_{A}}$ and data symbols $c$ we use, XOR will always exclude this situation. In other words, the hierarchical signals of this situation have always the same XC symbol. The configuration $(0,1,-1$,

$$
\left(\left\langle\mathbf{s}_{c_{A}}, \mathbf{s}_{c_{B}}\right\rangle,\left\langle\mathbf{s}_{c_{A}^{\prime}}, \mathbf{s}_{c_{B}^{\prime}}\right\rangle,\left\langle\mathbf{s}_{c_{A}}, \mathbf{s}_{c_{B}^{\prime}}\right\rangle,\left\langle\mathbf{s}_{c_{A}^{\prime}}, \mathbf{s}_{c_{B}}\right\rangle\right)=(0,0,-1,-1)
$$

Figure 25 One possible geometrical configuration of $\left(\left\langle\mathbf{s}_{c_{A^{\prime}}}, \mathbf{s}_{c_{B}}\right\rangle,\left\langle\mathbf{s}_{c_{A^{\prime}},}, \mathbf{s}_{c_{B^{\prime}}}\right\rangle,\left\langle\mathbf{s}_{c_{A^{\prime}}}, \mathbf{s}_{c_{B}{ }^{\prime}}\right\rangle\right)=(0,0,-1,-1)$. 
0 ) falls into the case $\mathbf{b}$ ), where the situation is geometrically possible, but it implies that $\left\|\mathbf{s}_{c_{A}}-\mathbf{s}_{c_{A^{\prime}}}\right\|^{2}=4$ and the condition (37) is fulfilled. The similar situation occurs for configurations

$$
\begin{gathered}
(0,1,0,-1),(0,-1,0,1),(0,-1,1,0), \\
(1,0,0,-1),(1,0,-1,0),(-1,0,0,1),(-1,0,1,0) .
\end{gathered}
$$

The case of $z=3$ are excluded in a very similar way by a) as for $z=1$. The last case $z=4$ have possible configurations

$$
(1,1,-1,-1),(-1,-1,1,1) .
$$

The former case means $\mathbf{s}_{c_{A}}=\mathbf{s}_{c_{B}} \& \mathbf{s}_{c_{A^{\prime}}}=\mathbf{s}_{c_{B}{ }^{\prime}}$ thus $k=l$ $\& m=n$, which corresponds to hierarchical signals from the main diagonal of XC matrix. This is excluded, because such a condition we already assume. The latter case implies $k=n \& m=l$ and is again excluded by symmetrical XC matrix.

By the above three steps, we have proved $z=0$, which proves the lemma.

\footnotetext{
Acknowledgements

This work was supported by the FP7-ICT SAPHYRE project, by Grant Agency of the Czech Republic grant 102/09/1624; and by Grant Agency of the Czech Technical University in Prague, grant SGS 10/287/OHK3/3T/13. Endnotes

${ }^{a}$ For the sake of notation simplicity, we assume orthonormal modulations rather than orthogonal with constant symbol energy. The results here shown for orthonormal modulation are true also in orthogonal case with constant symbol energy.
}

\section{Competing interests}

The authors declare that they have no competing interests.

Received: 28 January 2011 Accepted: 11 October 2011 Published: 11 October 2011

\section{References}

1. M Dohler, Y Li, Cooperative communications: Hardware, channel and PHY, (John Wiley \& Sons, 2010)

2. RW Yeung, Information Theory and Network Coding (Springer, 2008)

3. C Fragouli, D Katabi, A Markopoulou, M Medard, H Rahul, Wireless Network Coding: Opportunities and Challenges, in Military Communications Conference, 2007. MILCOM 2007. IEEE, 1-8 (2007)

4. P Popovski, H Yomo, The Anti-Packets Can Increase the Achievable Throughput of a Wireless Multi-Hop Network, in Communications, 2006. ICC' 06. IEEE International Conference on. 9, 3885-3890 (2006)

5. S Zhang, S Liew, P Lam, Physical Layer Network Coding, in ACM MOBICOM 2006, Los Angeles (2006)

6. J Sykora, A Burr, Hierarchical Alphabet and Parametric Channel Constrained Capacity Regions for HDF Strategy in Parametric Wireless 2-WRC, in Proc IEEE Wireless Commun Network Conf (WCNC), Sydney, Australia, 1-6 (2010)

7. J Sykora, A Burr, Network Coded Modulation with partial side-information and hierarchical decode and forward relay sharing in multi-source wireless network, in Wireless Conference (EW), 2010. European, 639-645 (2010)

8. T Koike-Akino, P Popovski, V Tarokh, Optimized constellations for two-way wireless relaying with physical network coding. Selected Areas in Communications, IEEE Journal on. 27(5), 773-787 (2009)

9. T Koike-Akino, P Popovski, V Tarokh, Adaptive Modulation and Network Coding with Optimized Precoding in Two-Way Relaying, in Global Telecommunications Conference, 2009. GLOBECOM 2009. IEEE 1-6 (2009)
10. T Uricar, J Sykora, Design Criteria for Hierarchical Exclusive Code with Parameter-Invariant Decision Regions for Wireless 2-Way Relay Channel. EURASIP Journal on Wireless Communications and Networking 2010, 1-13 (2010). [Article ID 921427]

11. R Krigslund, J Sorensen, P Popovski, T Koike-Akino, T Larsen, Physical Layer Network Coding for FSK Systems. IEEE Signal Process Lett (2009). [To appear]

12. M Valenti, D Torrieri, T Ferrett, Noncoherent physical-layer network coding using binary CPFSK modulation, in Military Communications Conference, 2009. MILCOM 2009. IEEE, 1-7 (2009)

13. O Simeone, U Spagnolini, Y Bar-Ness, S Strogatz, Distributed synchronization in wireless networks. Signal Processing Magazine, IEEE, 25(5), 81-97 (2008)

14. S Zhang, SC Liew, H Wang, Synchronization Analysis in Physical Layer Network Coding. CoRR (2010). abs/1001.0069

15. J Sykora, A Burr, Layered design of hierarchical exclusive codebook and its capacity regions for HDF strategy in parametric wireless 2-WRC. Accepted in IEEE Trans Veh Technol (2011)

16. J Sykora, A Burr, Wireless network coding: Network coded modulation in the network aware PHY layer, in Proc. IEEE Wireless Commun. Network. Conf (WCNC), Cancun, Mexico (2011). [Tutorial]

17. BD Mckay, IM Wanless, On the number of Latin squares. Ann Combin. 9 , 335-344 (2005). doi:10.1007/s00026-005-0261-7

18. T Uricar, Parameter-Invariant Hierarchical eXclusive Alphabet Design for 2WRC with HDF Strategy. Acta Polytechnica, 50(4), 79-86 (2010)

19. SM Kay, Fundamentals of Statistical Signal Processing: Detection Theory (Prentice-Hall, 1998)

20. S Benedetto, E Biglieri, Principles of digital transmission with wireless applications (New York, NY, USA: Kluwer Academic Publishers, 2002)

21. J Anderson, CE Sundberg, T Aulin, Digital Phase Modulation (Springer, 1986)

22. BE Rimoldi, A Decomposition Approach to CPM. IEEE Trans Inf Theory 34(2), 260-270 (1988). doi:10.1109/18.2634

23. F Amoroso, Pulse and Spectrum Manipulation in the Minimum (Frequency) Shift Keying (MSK) Format. Communications, IEEE Transactions on 24(3), 381-384 (1976). doi:10.1109/TCOM.1976.1093294

24. T Koike-Akino, P Popovski, V Tarokh, Denoising Strategy for ConvolutionallyCoded Bidirectional Relaying, in Proc IEEE Internat Conf on Commun (ICC) (2009)

doi:10.1186/1687-1499-2011-128

Cite this article as: Hekrdla and Sykora: Design of Uniformly Most Powerful Alphabets for HDF 2-Way Relaying Employing Non-Linear Frequency Modulations. EURASIP Journal on Wireless Communications and Networking 2011 2011:128.

\section{Submit your manuscript to a SpringerOpen ${ }^{\circ}$ journal and benefit from:}

- Convenient online submission

- Rigorous peer review

- Immediate publication on acceptance

- Open access: articles freely available online

- High visibility within the field

- Retaining the copyright to your article

Submit your next manuscript at $\gg$ springeropen.com 